\title{
Smad3 recruits the anaphase-promoting complex for ubiquitination and degradation of SnoN
}

\author{
Shannon L. Stroschein, ${ }^{1}$ Shirin Bonni, ${ }^{2}$ Jeffrey L. Wrana, ${ }^{2}$ and Kunxin Luo ${ }^{1,3}$ \\ ${ }^{1}$ Life Sciences Division, Lawrence Berkeley National Laboratory and Dept. of Molecular and Cell Biology, University \\ of California, Berkeley, California 94720, USA; ${ }^{2}$ Program in Molecular Biology and Cancer, Samuel Lunenfeld Research \\ Institute, Mount Sinai Hospital, Toronto, Ontario M5G 1X5, Canada
}

Smad proteins mediate transforming growth factor- $\beta$ (TGF- $\beta$ ) signaling to regulate cell growth and differentiation. SnoN is an important negative regulator of TGF- $\beta$ signaling that functions to maintain the repressed state of TGF- $\beta$ target genes in the absence of ligand. On TGF- $\beta$ stimulation, Smad3 and Smad2 translocate into the nucleus and induce a rapid degradation of SnoN, allowing activation of TGF- $\beta$ target genes. We show that Smad2- or Smad3-induced degradation of SnoN requires the ubiquitin-dependent proteasome and can be mediated by the anaphase-promoting complex (APC) and the UbcH5 family of ubiquitin-conjugating enzymes. Smad3 and to a lesser extent, Smad2, interact with both the APC and SnoN, resulting in the recruitment of the APC to SnoN and subsequent ubiquitination of SnoN in a destruction box (D box)-dependent manner. In addition to the $\mathrm{D}$ box, efficient ubiquitination and degradation of SnoN also requires the Smad3 binding site in SnoN as well as key lysine residues necessary for ubiquitin attachment. Mutation of either the Smad3 binding site or lysine residues results in stabilization of SnoN and in enhanced antagonism of TGF- $\beta$ signaling. Our studies elucidate an important mechanism and pathway for the degradation of SnoN and more importantly, reveal a novel role of the APC in the regulation of TGF- $\beta$ signaling.

[Key Words: SnoN; TGF- $\beta$; Smad proteins; anaphase-promoting complex; ubiquitin; degradation]

Received May 18, 2001; revised version accepted August 16, 2001.

Smad proteins are critical components of the TGF- $\beta$ signaling pathways. In the absence of TGF- $\beta$, the two highly homologous receptor-associated Smads, Smad2 and Smad3, are distributed mostly in the cytoplasm. On ligand binding, the activated type I TGF- $\beta$ receptor serine/threonine kinase (T $\beta R I)$ phosphorylates these Smad proteins, allowing them to translocate into the nucleus and form heteromeric complexes with the common-mediator Smad4 (Heldin et al. 1997; Massague et al. 2000; Chacko et al. 2001; Wu et al. 2001). In the nucleus, the Smad complexes interact with various cellular partners and participate in diverse downstream activities. The Smads can bind to the TGF- $\beta$-responsive promoter DNA either directly through the N-terminal Mad homology-1 (MH1) domain (Massague et al. 2000) or in conjunction with other sequence-specific DNA binding proteins such as the FAST and Milk family of proteins (Chen et al. 1996, 1997; Liu et al. 1997a; Labbe et al. 1998; Zhou et al. 1998b; Germain et al. 2000). Through the C-terminal

${ }^{3}$ Corresponding author.

E-MAIL kluo@lbl.gov; FAX (510) 486-6488.

Article and publication are at http://www.genesdev.org/cgi/doi/10.1101/ gad.912901.
MH2 domains, Smad proteins interact with general or promoter-specific transcriptional activators to activate transcription of various TGF- $\beta$ target genes (Massague and Wotton 2000). They may also associate with transcriptional corepressors such as TGIF (Wotton et al. 1999), Ski (Akiyoshi et al. 1999; Luo et al. 1999; Sun et al. 1999a), SnoN (Stroschein et al. 1999b; Sun et al. 1999b), and SNIP1 (Kim et al. 2000). Some activated Smads can also be targeted for degradation by the ubiquitin-dependent proteasome (Lo and Massague 1999; Zhu et al. 1999; Lin et al. 2000; Zhang et al. 2001).

Among the negative regulators of Smad function, SnoN and Ski are two closely related members of the Ski family of nuclear proto-oncoproteins. When overexpressed, they cause oncogenic transformation of chicken and quail embryo fibroblasts as well as muscle differentiation of quail embryo cells (Colmenares and Stavnezer 1989; Colmenares et al. 1991; Boyer et al. 1993). High levels of Ski or SnoN are detected in many types of human cancer cells (Nomura et al. 1989; Fumagalli et al. 1993). Interestingly, mice lacking one copy of the sno gene were also found to be more susceptible to chemical carcinogens (Shinagawa et al. 2000). Thus, the oncogenic potential of SnoN appears to be related to deregulation of 
its normal expression levels. Human SnoN is a ubiquitously expressed transcriptional corepressor of 684 amino acids (Nomura et al. 1989) that interacts with Smad2, Smad3, and Smad4 to antagonize TGF- $\beta$ signaling (Stroschein et al. 1999b; Sun et al. 1999b). We have shown that SnoN may function to maintain the repressed state of TGF- $\beta$ target genes in the absence of TGF- $\beta$ and may also participate in negative feedback control of TGF- $\beta$ signaling (Stroschein et al. 1999b). On TGF- $\beta$ stimulation, a rapid degradation of SnoN occurs, most likely mediated by Smad3 and to a lesser extent, Smad2. The removal of the inhibitory SnoN may be crucial for activation of TGF- $\beta$ signaling as this allows the Smads to activate transcription of TGF- $\beta$ responsive genes.

We investigated the molecular mechanism by which Smad3 and Smad2 induce degradation of SnoN. Degradation of most intracellular proteins is mediated by the proteasome, which is an essential component of the ATP-dependent proteolytic pathway and is present in the nucleus and cytosol of all eukaryotic cells (Coux et al. 1996). Most proteins degraded by the $26 \mathrm{~S}$ proteasome require prior polyubiquitination. Ubiquitination occurs through a stepwise action of three classes of enzymes (Varshavsky 1997; Hershko and Ciechanover 1998). Initially, ubiquitin is activated by the E1 ubiquitin-activating enzyme and transferred to the E2 ubiquitin conjugating (Ubc) enzymes. In the presence of an E3 ubiquitin ligase, the ubiquitin is attached covalently to a particular lysine(s) in the substrate. The cycle repeats to form a polyubiquitin chain, leading to the recognition and degradation of the target protein by the proteasome.

The E3 ubiquitin ligases often interact with the substrates in a regulated manner and play central roles in determining the specificity of ubiquitination. Among the E3s, the HECT (homology to E6-AP $\underline{\mathrm{C}}$ terminus)domain-containing E3s and RING-finger-containing E3s have been characterized extensively in mammalian cells. The HECT-domain E3s are characterized by the ability to form a thiolester intermediate with the activated ubiquitin before transferring the ubiquitin to the substrates (Scheffner et al. 1993; Huibregtse et al. 1995). One HECT-domain E3 family, the Smurf family, has been shown to interact with the PY motifs present in the N-terminal and linker regions of the Smads and mediates degradation of TGF- $\beta$-signaling molecules (Zhu et al. 1999; Kavsak et al. 2000; Lin et al. 2000; Bonni et al. 2001; Zhang et al. 2001). For example, Smurf2, through interaction with Smad7, can be recruited to the TGF- $\beta$ receptors, leading to their degradation (Kavsak et al. 2000). By interacting with Smad2, Smurf2 can also be recruited to SnoN to induce its degradation (Bonni et al. 2001). High levels of Smurf2 have also been shown to degrade Smad1 (Zhang et al. 2001) and Smad2 (Lin et al. 2000) in transfected cells. In contrast to the HECT-domain E3s, the RING-finger-containing E3s do not form a covalent bond with the activated ubiquitin. Many RING-finger-containing E3s are part of a large protein complex, such as the anaphase-promoting complex (APC) or SCF (Skp1-cullin-F-box protein), that also contain other subunits required for substrate recognition and recruitment and for regulation of the E3 ligase activity. The APC is a cell-cycle regulated ubiquitin-protein ligase that consists of 11 core subunits in mammalian cells (Gmachl et al. 2000), one of which, APC11, is the RING-finger-containing subunit with the E3 ligase activity (Gmachl et al. 2000; Leverson et al. 2000). Two WD-40 proteins, CDC20 and CDH1, can associate with the APC and activate its activity in a substrate-specific and cell cycle-dependent manner (Peters 1999; Zachariae and Nasmyth 1999). CDC20 interacts with the APC during mitosis and targets proteins containing a destruction box (D box), such as cyclin B and Pds1, for degradation (Fang et al. 1998; Lorca et al. 1998; Shirayama et al. 1999; Kramer et al. 2000; Rudner and Murray 2000). CDH1, present in $\mathrm{G}_{1}$ (Fang et al. 1998) and also in differentiated cells (Gieffers et al. 1999), can recognize the D box or KEN box motifs present in cell-cycle-specific as well as noncell-cycle proteins and activate the APC for their ubiquitination (Pfleger and Kirschner 2000). Thus, the APC can function in both proliferating and differentiated cells and may regulate the degradation of both cell-cycle and noncell-cycle proteins.

We examined the mechanism of TGF- $\beta$ - and Smaddependent turnover of SnoN and have identified key components of the ubiquitin machinery involved in the process. Our studies suggest that the APC is involved in the Smad3-induced ubiquitination and degradation of SnoN. Because the transforming activity of SnoN is closely correlated with its high steady-state level, the elucidation of mechanisms regulating the intracellular level of SnoN may provide critical insights into the cause of oncogenic transformation by SnoN.

\section{Results}

TGF- $\beta$ - and Smad3-induced degradation of SnoN is mediated by the ubiquitin-dependent proteasome

We have shown previously that TGF- $\beta$ induces a rapid degradation of SnoN (Stroschein et al. 1999b). This activity appears to be mediated by Smad3 and to a slightly lesser extent, Smad2, because overexpression of Smad2 or Smad3 resulted in degradation of SnoN even in the absence of TGF- $\beta$ signaling (Fig. 1A). It is known that overexpressed Smad 2 and Smad 3 can partially bypass the requirement for ligand and localize in the nucleus, oligomerize, and activate downstream events (Wu et al. 1997; Zhang et al. 1997). Activation of TGF- $\beta$ signaling can further enhance these events. This property of the Smads allows us to investigate directly the effects of Smad proteins on degradation of SnoN.

To investigate the mechanism responsible for Smad3induced degradation of SnoN, we first employed inhibitors of various intracellular degradation machineries in pulse-chase assays. ALLM, an inhibitor of $\mathrm{Ca}^{2+}$-activated proteases (calpains), had no effect on Smad3-induced degradation of SnoN (Fig. 1B). In contrast, lactacystin, an 
Stroschein et al.

Figure 1. Smad3-and TGF- $\beta$-induced degradation of SnoN is mediated by the ubiquitin-dependent proteasome. (A) Pulse-chase analysis of SnoN degradation by various Smad proteins. 293T cells cotransfected with HA-SnoN and the indicated Flagtagged Smads were subjected to pulse-chase assays as described in Materials and Methods. Immunoprecipitation was carried out with anti-Flag to purify Smad-associated SnoN or with anti-HA to purify total cellular HA-SnoN. The half-lives of the two populations were similar. As a control, SnoN from singly transfected cells was isolated by immunoprecipitation with anti-HA. Abbreviations used: S3, Smad3; S3C, MH2 domain of Smad3; S3N, MH1 domain of Smad3; S2, Smad2; S2C, MH2 domain of Smad2; S2NL, MH1 and linker domains of Smad2; S4, Smad4. (B) Proteasome inhibitors block Smad3-induced degradation of SnoN. Transfected 293T cells were pretreated with DMSO (control) or various proteolytic inhibitors for 45 min followed by a pulse-chase assay as described in Materials and Methods. Inhibitors were present throughout the duration of the pulsechase. Quantification of ${ }^{35}$ S-labeled SnoN was carried out with the Bio-Rad Molecular Imager FX system and is shown below the gels. $(C)$ Lactacystin blocks TGF- $\beta$-induced degradation of endogenous SnoN. Hep3B cells were pretreated with DMSO (lanes 1-3) or lactacystin (lanes 4-6) for $4 \mathrm{~h}$ followed by stimulation with 200 pM TGF- $\beta 1$ for the indicated times. Endogenous SnoN was isolated by immunoprecipitation with an anti-SnoN anti-serum and analyzed by immunoblotting with anti-SnoN. (D) Smad2 and Smad3 induce ubiquitination of SnoN. HA-Smads or Flag-SnoN were transfected either alone or together into 293T cells and isolated by immunoprecipitation with either anti-HA (lanes 1-3) or anti-Flag (lanes 4-7). Polyubiquitination of SnoN was detected by immunoblotting of the immunoprecipitates with an antiubiquitin antibody (top). The amounts of SnoN (middle) and Smads present in the immunoprecipitates were shown on the two lower panels. $(E)$ The $\mathrm{MH} 2$ domain of Smad3 induces SnoN ubiquitination. The experiments were carried out as described in $D$.
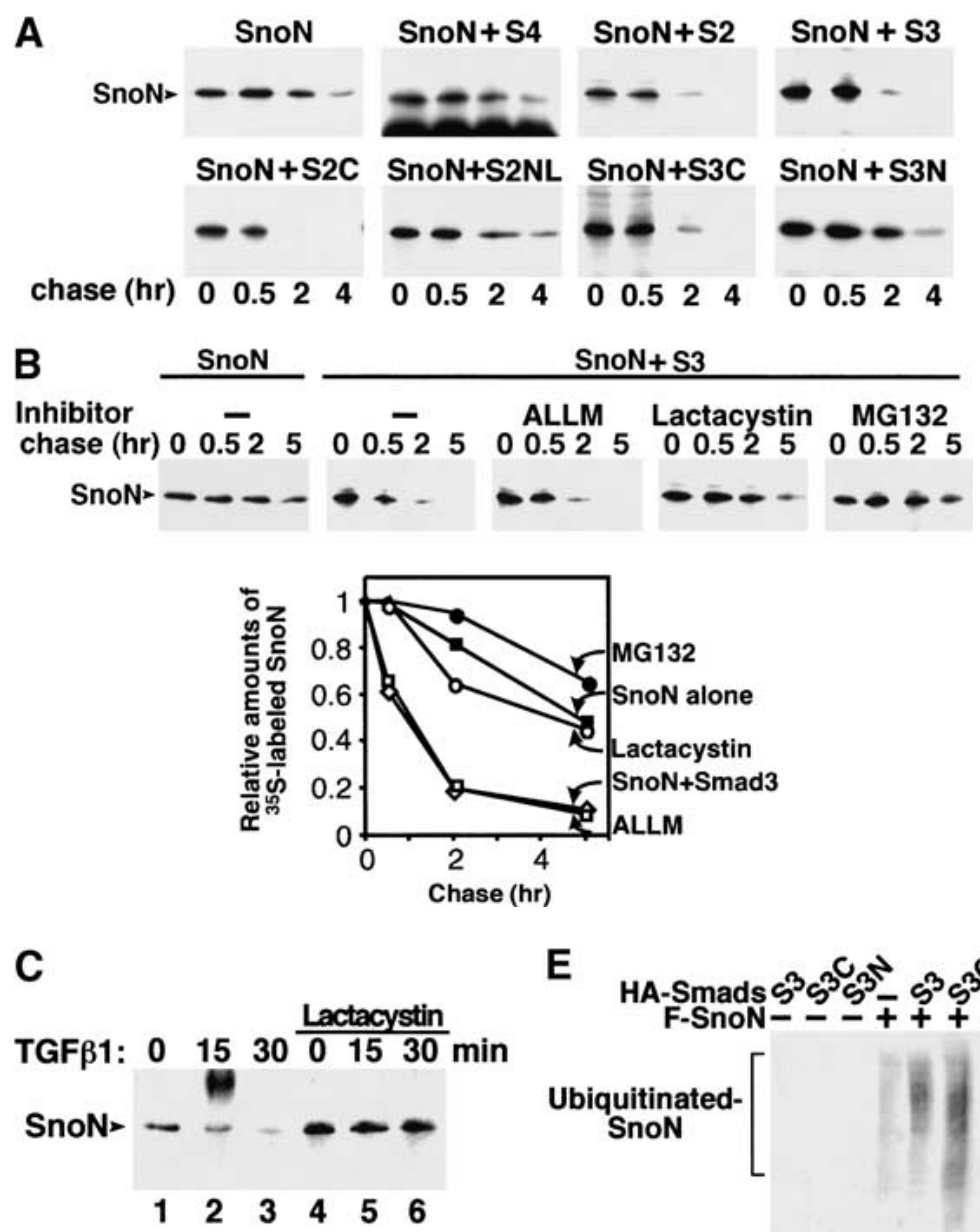

D
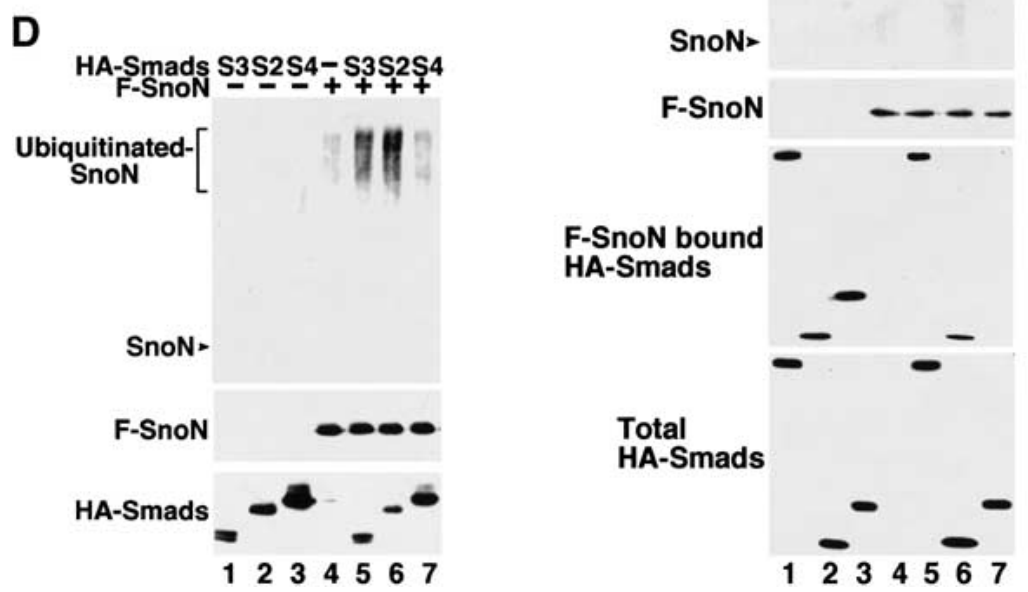

inhibitor specific for the proteasome, and MG132, a proteasome and calpain inhibitor, blocked SnoN degradation (Fig. 1B). Furthermore, treatment of Hep3B cells with lactacystin also prevented TGF- $\beta$-induced degradation of endogenous SnoN proteins (Fig. 1C), suggesting that TGF- $\beta$-induced, Smad3-dependent degradation of SnoN is mediated by the proteasome.

Because most proteins degraded by the proteasome require ubiquitination (Hershko and Ciechanover 1998), we asked whether Smad2 and Smad3 induce ubiquitina- tion of SnoN. SnoN was isolated from transfected cells by immunoprecipitation with an anti-SnoN antiserum and detected by immunoblotting with anti-ubiquitin. In the absence of Smad proteins, SnoN exhibited a low level of polyubiquitination (Fig. 1D, lane 4). Coexpression of SnoN with Smad3 or Smad2, but not with Smad4, resulted in a marked increase in the polyubiquitination of SnoN (Fig. 1D, lanes 5-7). This is consistent with the observation that Smad3 and Smad2, but not Smad4, induced degradation of SnoN (Fig. 1A). 
A direct interaction between Smad3 and SnoN is necessary but not sufficient for the degradation of SnoN

The ubiquitination and degradation of SnoN are mediated by the MH2 domains of Smad2 and Smad3 (Smad2C and Smad3C) (Fig. 1A,E), but not by the N-termini and linker regions $(\mathrm{Smad} 3 \mathrm{~N}$ and $\mathrm{Smad} 2 \mathrm{NL})$ that do not interact with SnoN. To further define the region in Smad2 and Smad3 required for binding and degradation of $S n o N$, C-terminal deletions of Smad3 and chimeras between Smad1 and Smad2 were tested in binding assays (Fig. 2A,B). All Smad3 deletion mutants bound SnoN, albeit weakly (Fig. 2A, lanes 5-8). This suggests that the SnoN
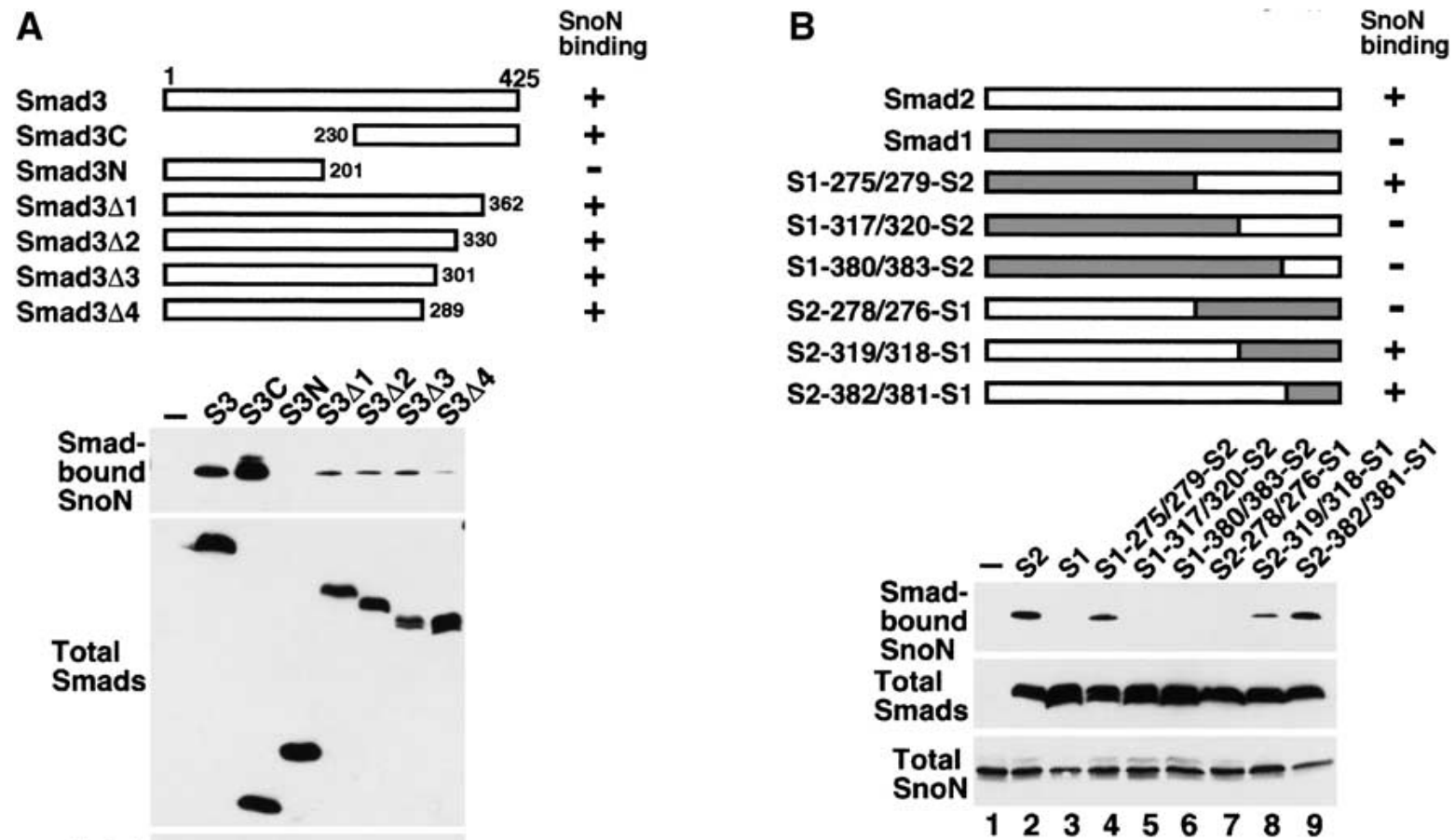

Total $\Rightarrow-\infty---$

$\begin{array}{llllllll}1 & 2 & 3 & 4 & 5 & 6 & 7 & 8\end{array}$
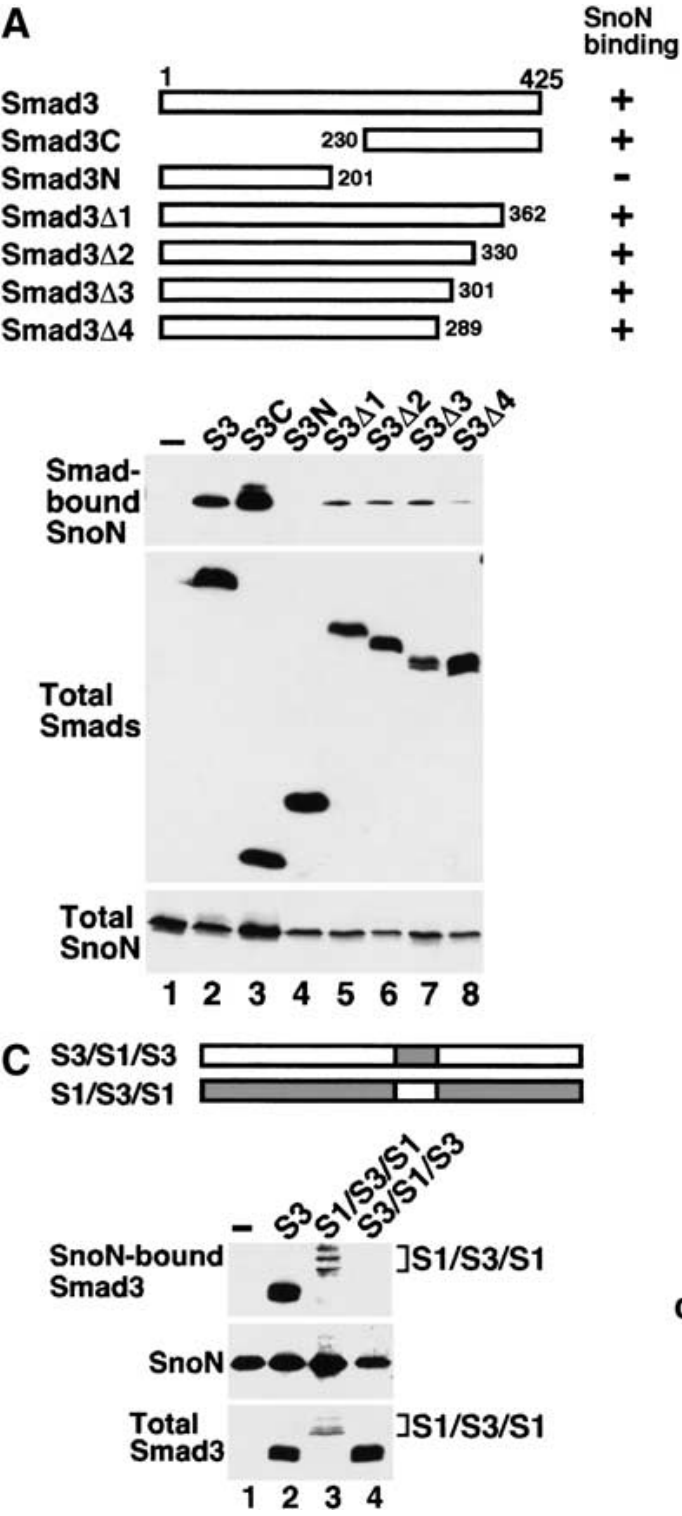

$\begin{array}{lllllllll}1 & 2 & 3 & 4 & 5 & 6 & 7 & 8 & 9\end{array}$

$\begin{array}{lll}2 & 3 & 4\end{array}$

Figure 2. A direct interaction between Smad3 and SnoN is necessary but not sufficient for degradation of SnoN. $(A)$ Residues $230-289$ of Smad3 are required for interaction with SnoN. Flag-tagged full-length or truncated Smad3 was cotransfected into $293 \mathrm{~T}$ cells together with HA-SnoN and isolated by immunoprecipitation with anti-Flag agarose. The immunoprecipitates were subjected to immunoblotting with an anti-HA antibody to detect SnoN that bound to Smad3 (top) or with anti-Flag to control for Smad levels (middle). Cell lysates were blotted with anti-HA to control for SnoN expression (bottom). (B) Residues 279-318 of Smad2 are required for interaction with SnoN. Flag-tagged Smad1, Smad2, or Smad1/Smad2 chimeras were cotransfected together with HA-SnoN and analyzed as described in $A$. (C) Residues 237-276 of Smad3 are necessary but not sufficient for degradation of SnoN. (Left) Binding assay. Flag-SnoN was transfected either alone or together with HA-tagged Smads and isolated by immunoprecipitation with anti-Flag antibodies followed by Western blotting with anti-HA to detect Smads that bound to SnoN (top) or with anti-Flag to detect SnoN (middle). Cell lysates were blotted with anti-HA to control for Smad expression (bottom). (Right) Pulse-chase assay. 293T cells cotransfected with HA-SnoN and Flag-Smad were subjected to pulse-chase assay. (S3/S1/S3) Smad3 residues 1-236 and 277-425, and Smad1 residues 276-317. (S1/S3/S1) Smad1 residues 1-275 and 318-465, and Smad3 residues 237-276. 
binding site lies between amino acid residues 230 and 289. The weak binding is most likely attributable to the inherent instability of these deletions compared to the wild-type Smad3 (Fig. 2A; data not shown). To map the SnoN binding site in Smad2, Smad1/Smad2 chimeras were employed. Because Smad1 does not bind to SnoN (Fig. 2B, lane 3), any binding to SnoN is contributed by the Smad2 portion of the molecule. Chimeras containing amino acids 279-319 from Smad2 (equivalent to residues 237-276 in Smad3) bound to SnoN (Fig. 2B, lanes 4,8,9), whereas those containing the equivalent region from Smad1 did not (Fig. 2B, lanes 5-7). Thus, the equivalent region in Smad3 (residues 230-289) and in Smad2 (residues 279-319) mediates binding to SnoN.

We next tested whether this region is necessary and sufficient for degradation of SnoN. Because deletion of sequences in the MH2 domains of the Smads may affect the folding and stability of the proteins, we employed two Smad3/Smad1 chimeras in this experiment, one (S3/ S1/S3) with the SnoN binding domain of Smad3 replaced with the Smad1 sequence (Smad3 residues $1-236$ and 277-425, Smad1 residues 276-317) and another (S1/S3/ S1) with only the SnoN binding domain of Smad3 (Smad1 residues 1-275 and 318-465, Smad3 residues 237-276). S3/S1/S3 was unable to bind SnoN and did not induce ubiquitination (Fig. 2C; data not shown) or degradation of SnoN (Fig. 2C). S1/S3/S1, on the other hand, bound SnoN. However, this chimera was unable to induce degradation of SnoN to the same extent as wildtype Smad3 (Fig. 2C), suggesting that amino acid residues 237-276 in Smad3 are sufficient for binding to SnoN, but not for SnoN degradation. Interaction of Smad3 with other cellular factors, presumably the ubiquitination machinery, is also required for efficient degradation of SnoN (see Fig. 6A, below). In this sense, Smad3 or Smad2 serves as the targeting subunit of an E3 ligase complex.

\section{Domains in SnoN required for ubiquitination and degradation}

At least two determinants are required for a protein to be ubiquitinated and degraded by the proteasome: recognition site(s) for the targeting subunit(s) of an E3 ubiquitin ligase and a lysine residue(s) that serves as a ubiquitin attachment site (Hershko and Ciechanover 1998). Deletion mutants of SnoN were generated to map these two determinants. Deletion of the first 96 amino acids (SnoN 97-684) disrupted binding to Smad3 (Stroschein et al. 1999b; Fig. 3B). Within this region, amino acid residues 89-92 appeared to be required for binding to Smad3 as substitutions of these residues with alanine (SnoN 8992A) disrupted binding to Smad2 or Smad3 (Fig. 3B, third panel from the top; J. He and K. Luo, in prep.). Neither the deletion mutant nor the point mutant was ubiquitinated (Fig. 3B) or degraded in response to Smad3 coexpression (Fig. 3C). Thus, interaction with Smad2 or Smad3 is required for ubiquitination and degradation of SnoN. When expressed in the TGF- $\beta$-responsive Hep3B

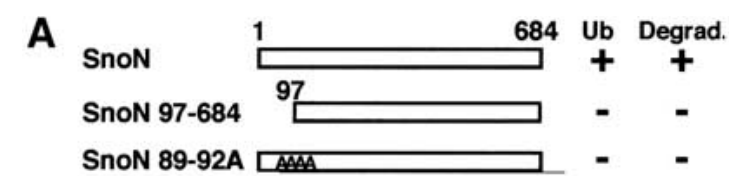

B
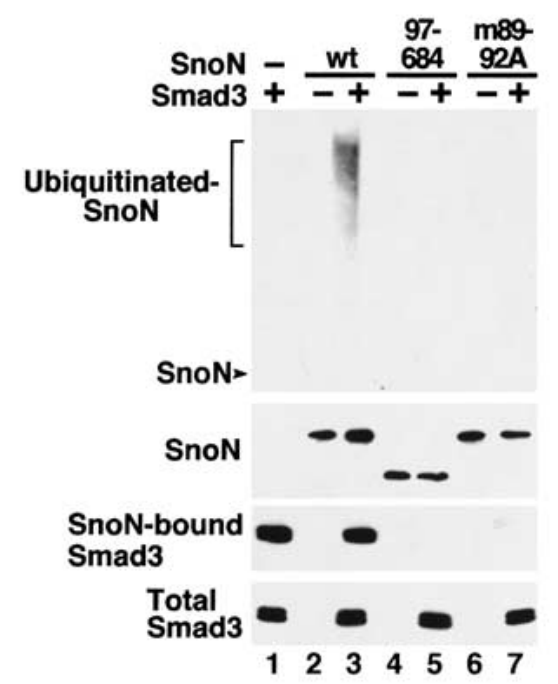

C
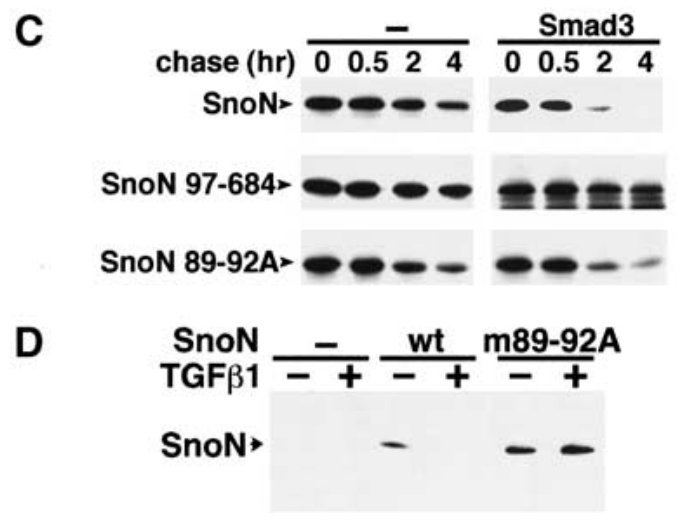

Figure 3. Smad2- or Smad3-binding site in $\mathrm{SnoN}$ is required for the ubiquitination and degradation of SnoN. $(A)$ SnoN mutants. (B) Ubiquitination of SnoN requires binding to $S \operatorname{mad} 2$ or $S$ mad3. HA-Smad3 or Flag-SnoN was transfected either individually or together into $293 \mathrm{~T}$ cells. Flag-SnoN was isolated by immunoprecipitation with anti-Flag (lanes 2-7) antibody followed by Western blotting with anti-ubiquitin (top), anti-Flag (second panel), or anti-HA (third panel). As a control, HA-Smad3 was isolated from singly transfected cells by immunoprecipitation with anti-HA (lane 1). Total Smad levels were detected by immunoblotting of the cell lysates with anti-HA (bottom). (C) Pulse-chase assay. 293T cells transfected with HA-SnoN and Flag-Smad3 were subjected to the pulse-chase assay as described in Materials and Methods. SnoN was isolated from the transfected cells by immunoprecipitation with the anti-HA antibody. (D) TGF- $\beta$-induced degradation of SnoN is mediated by the R-Smads. Hep3B cells were transiently transfected with Flag-tagged wild-type or mutant SnoN constructs. Cells were treated with TGF- $\beta$ for $30 \mathrm{~min}$, and SnoN was isolated by immunoprecipitation with anti-Flag agarose and detected by Western blotting with anti-Flag antiserum.

cells, SnoN 89-92A also failed to be degraded in response to TGF- $\beta$ (Fig. $3 \mathrm{D})$, again confirming that TGF- $\beta$-induced 
degradation of SnoN is mediated by interaction with Smad2 and Smad3.

We have shown previously that a mutant SnoN containing only the N-terminal 366 amino acids (SnoN 1-366) can not be degraded by the coexpression of Smad3 (Stroschein et al. 1999b). Because this mutant still binds Smad3, an additional element required for SnoN degradation must be present in the carboxyl half of SnoN between residues 367-684. In the ubiquitination assay, this mutant failed to be ubiquitinated in the presence of Smad3 (data not shown). Thus, the lysine residues required for ubiquitination may be located in the carboxyl half of SnoN and are missing in SnoN 1-366. Serial deletions from the $\mathrm{C}$ terminus of SnoN were made, and these deletion mutants were tested for their ability to be degraded by Smad3 coexpression. Because some SnoN truncations have similar molecular mass with Smad3 and therefore are difficult to resolve on an SDS-PAGE gel, Smad2C was used in the pulse-chase assay to induce degradation of SnoN. Deletions up to residue 459 (SnoN 1-459) had no effect on Smad2C-induced degradation of SnoN (Fig. 4B; data not shown). This mutant was degraded by the coexpression of Smad2C with kinetics similar to that of wild-type SnoN (Fig. 4B). Thus, the lysine residue(s) must reside between residues 367-459.
There are eight lysine residues within this region (Fig. 4A). These eight residues were mutated individually or in various combinations to arginine or alanine, and the resulting mutants tested in ubiquitination and degradation assays. Alteration of the first five lysines (Lys 383, 407, 423, 427, 432), SnoN K1-5R, had no effect on Smad3-induced ubiquitination (Fig. 4C). In contrast, mutation of the last three lysines, Lys 440, 446, and 449, either alone (SnoN K6R, K7-8R) or in combination with other lysine residues (SnoN K6-8R, K1-6R) abolished Smad3-induced ubiquitination (Fig. 4C). Consistent with this, mutant SnoN lacking any of the three lysine residues can not be degraded by TGF- $\beta$ when transfected into Hep3B cells (Fig. 4D). Thus lysines 440, 446, and 449 are important for both ubiquitination and degradation of SnoN.

\section{The UbcH5 family of E2 ubiquitin conjugating enzymes may be required for Smad3-induced SnoN degradation}

To identify the cellular machinery responsible for SnoN ubiquitination and degradation, various dominant-negative E2 ubiquitin conjugating enzymes were tested for their ability to block Smad3-induced degradation of
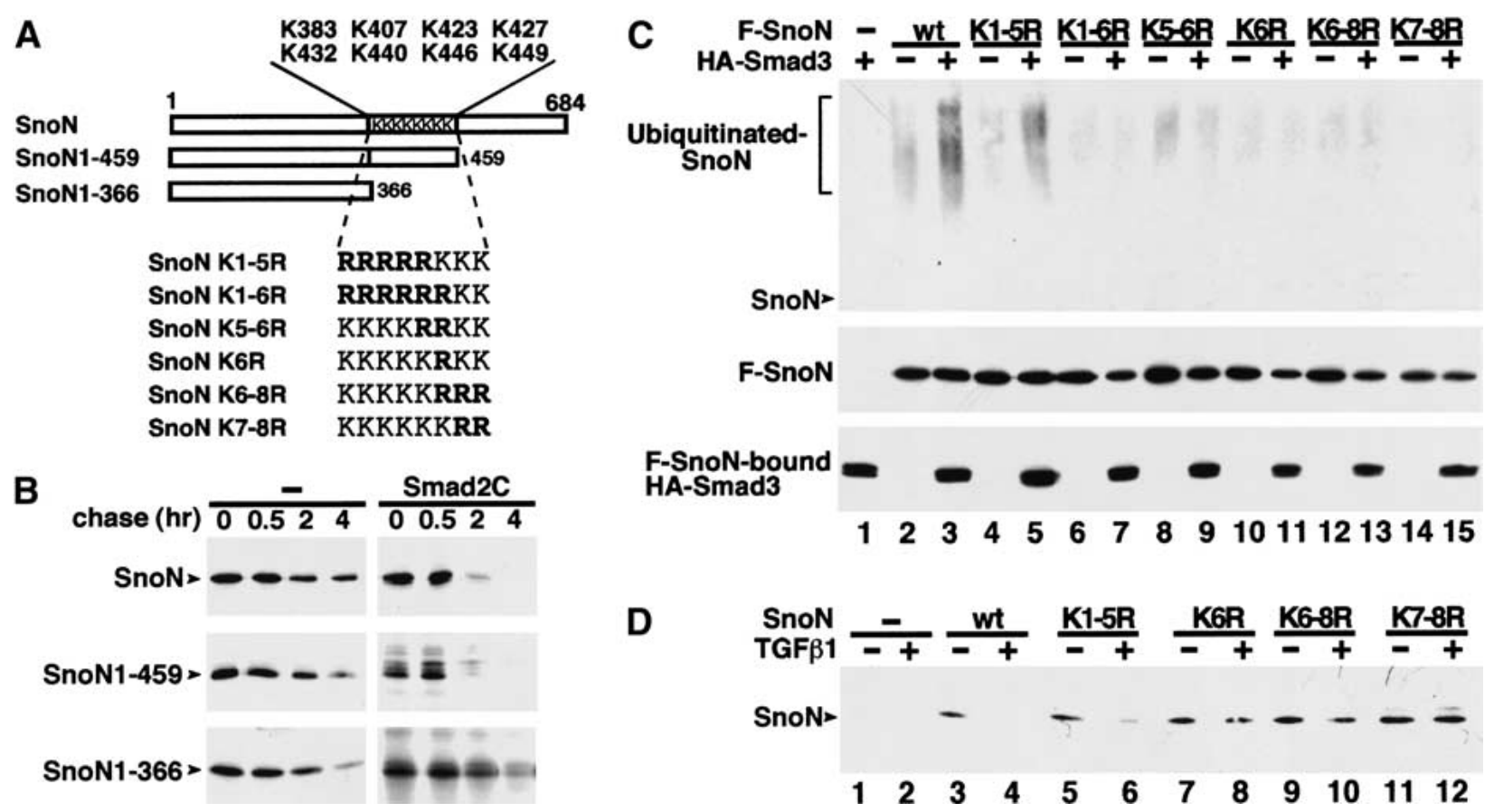

Figure 4. Lysines 440, 446, and 449 of SnoN are required for ubiquitination. (A) SnoN deletions and point mutations. Lysine residues were mutated to arginine and numbered as follows: 1, K383; 2, K407; 3, K423; 4, K427; 5, K432; 6, K440; 7, K446; and 8, K449. (B) Pulse-chase assay of the SnoN deletion mutants. 293T cells were transfected with HA-SnoN and Flag-Smad2C and subjected to the pulse-chase assay. SnoN associated with Smad2C was isolated by immunoprecipitation with the anti-Flag antibody and detected by autoradiography. (C) Mutation of lysines 440, 446, and 449 disrupts Smad3-induced ubiquitination of SnoN. Flag-SnoN was cotransfected together with HA-Smad3 and isolated by immunoprecipitation with anti-Flag (lanes 2-15) followed by Western blotting with anti-ubiquitin (top), anti-Flag (middle) or anti-HA antibodies (bottom). As a control, HA-Smad3 was isolated from singly transfected cells by immunoprecipitation with anti-HA (lane 1). (D) Mutation of lysines 440, 446, and 449 blocks TGF- $\beta$-induced degradation of SnoN. Hep3B cells were transiently transfected with the indicated Flag-tagged SnoN constructs. Cells were treated with TGF- $\beta$ for 30 min, and SnoN was isolated by immunoprecipitation with anti-Flag agarose and detected by Western blotting with anti-Flag antiserum. 
Stroschein et al.

SnoN in pulse-chase assays. These mutant E2 enzymes contain catalytic site mutations and have been shown to behave in a dominant negative manner (Gonen et al. 1999). Cotransfection of dominant-negative mutants of the closely related $\mathrm{UbcH} 5 \mathrm{a}, \mathrm{UbcH} 5 \mathrm{~b}$, or UbcH5c inhibited Smad3-induced SnoN degradation (Fig. 5A). In contrast, dominant negative UbcH6, UbcH7, UbcH8, UbcH9, and UbcH10 had no effect on the half-life of SnoN in the presence of Smad3 (Fig. 5A), even though all these mutant E2s were expressed well in transfected cells (data not shown). This suggests that the UbcH5 family of E2 enzymes may mediate Smad3-induced degradation of SnoN. Because the UbcH5 family of E2s has been shown to function in concert with both the HECTdomain E3 ubiquitin ligase E6-AP and the APC or cyclosome that targets the D box motif, ubiquitination of SnoN may involve members of these ubiquitin ligase families (Scheffner et al. 1994; Jensen et al. 1995; Rolfe et al. 1995; Gmachl et al. 2000; Leverson et al. 2000).

\section{Smad3-induced degradation of SnoN is mediated by a $D$ box-dependent ubiquitination pathway}

We have shown recently that Smurf2, a HECT-domain E3 ubiquitin ligase, can be recruited to SnoN through interaction with Smad2 or Smad3 in a TGF- $\beta$-dependent manner to mediate degradation of SnoN (Bonni et al. 2001; data not shown). This interaction requires the PY motif in the $\mathrm{MH} 1 /$ linker regions of Smad2 or Smad3 (Bonni et al. 2001; data not shown). However, Smurf2 may not be the only E3 ligase responsible for Smad3induced degradation of SnoN, as a mutant Smad3 lacking the PY motif (Smad3 $\Delta \mathrm{PY}$ ) still induced ubiquitination and degradation of SnoN (Fig. 5B), albeit less efficiently
A

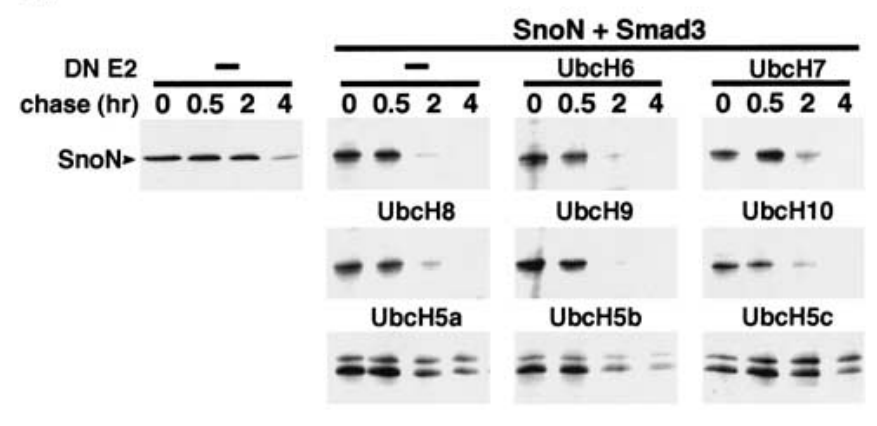

B

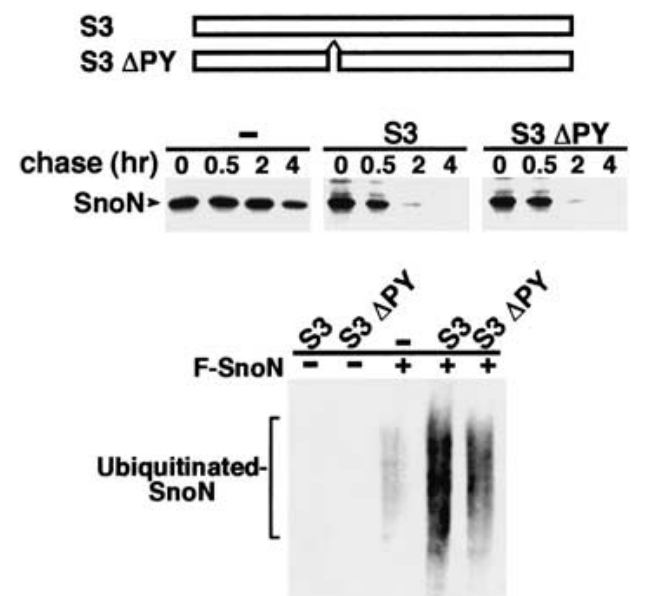

C

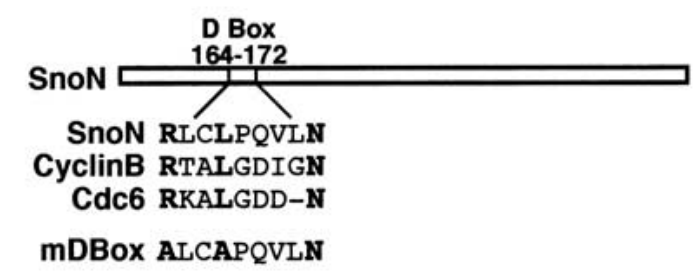

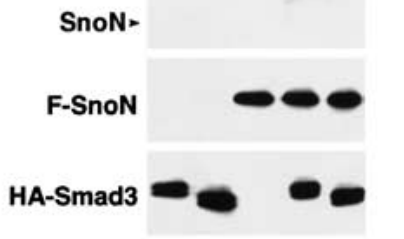

$\begin{array}{lllll}1 & 2 & 3 & 4 & 5\end{array}$

Figure 5. Ubiquitination pathways and components involved in TGF- $\beta$-induced degradation of SnoN. (A) The UbcH5 family of E2 ubiquitin-conjugating enzymes are required for SnoN degradation. Dominant negative forms of various E2 enzymes were cotransfected into $293 \mathrm{~T}$ cells together with Smad3 and SnoN. The ability of Smad3 to induce degradation of SnoN was analyzed by pulse-chase assay as described in Materials and Methods. $(B)$ Smad3-induced ubiquitination and degradation of SnoN does not require the PY motif of Smad3. (Top) Pulse-chase assay. 293T cells were transfected with SnoN and the Smad3 constructs as indicated and subjected to pulse-chase assays. (Bottom) Ubiquitination assay. HA-Smad3 or Flag-SnoN constructs were transfected either individually or together into 293T cells and isolated by immunoprecipitation with anti-HA (lanes 1,2) or anti-Flag (lanes 3-5) antibodies followed by Western blotting with anti-ubiquitin (top), anti-Flag (middle), or anti-HA (bottom). (C) Smad3-induced ubiquitination and degradation of SnoN requires the D box sequence. (Top) Alignment of various D box sequences. (Middle) Pulse-chase assay. (Bottom) Ubiquitination assay. The experiments were carried out as in $B$. 
than the wild-type Smad3, even though this Smad3 3 PY was unable to bind to Smurf2 (data not shown). Furthermore, the MH2 domains of Smad3 and Smad2, which do not contain the PY motifs, can induce ubiquitination and degradation of SnoN (Fig. 1A). Thus, an additional Smurf2-independent ubiquitination pathway can be recruited by Smad3 and Smad2 to induce degradation of SnoN. Activation of both pathways may be required for maximal degradation of SnoN.

A D box motif was found in SnoN between residues 164-172 that shows a consensus of RxxLxxxxN (Fig. 5C; Y. Wan, X. Liu, and M.W. Kirschner, in prep.). This motif, targeted by the APC, is usually found in cyclins and other cell cycle-regulated proteins and mediates degradation of these proteins (Glotzer et al. 1991; Peters 1999). To determine whether the $\mathrm{D}$ box is required for Smad3induced degradation of SnoN, two critical residues in the $\mathrm{D}$ box were mutated, and the resulting SnoN mutant was tested in the pulse-chase assay. As shown in Figure 5C, mutation of the D box (mDbox) prevented Smad3-induced ubiquitination and degradation of SnoN. Thus, the $\mathrm{D}$ box is essential for Smad3-induced degradation of SnoN, and a ubiquitin ligase complex that targets the D box, possibly the APC, is required for Smad3-induced degradation of SnoN.

\section{Smad3 and Smad2 interact with the APC to induce ubiquitination of SnoN}

To investigate whether the APC can be recruited by Smad3 and Smad2 to induce degradation of SnoN, we first examined the ability of the Smads to interact with the APC components in the nucleus by a coimmunoprecipitation assay. In nuclear extracts prepared from 293T cells transfected with Flag-Smad3, Flag-Smad3 was found to complex with endogenous Cdc27 and Cdc16, two important components of the APC (Peters 1999; Zachariae and Nasmyth 1999), and this interaction was mediated by the C-terminal MH2 domain of Smad3 (Fig. $6 \mathrm{~A})$. Activation of TGF- $\beta$ signaling can further enhance this interaction (Fig. 6C). The APC-binding site in the Smad3 MH2 domain is outside the SnoN-binding region because the S1/S3/S1 chimera, which is sufficient for binding to SnoN, failed to interact with the APC (Fig. 6A, lane 81, whereas the S3/S1/S3 did not bind to SnoN, but associated with the APC (Fig. 6A, lane 7). Because neither chimera induced efficient degradation of SnoN, binding of Smad3 to both SnoN and the APC may be necessary for ubiquitination of SnoN. In addition to Smad3, Smad2 but not Smad4 also formed a complex with endogenous APC (Fig. 6A), albeit to a lesser extent, consistent with the fact that only Smad3 and Smad2, but not Smad4, induce degradation of SnoN.

$\mathrm{CDH} 1$, the targeting subunit of the APC, recognizes the D box or KEN box motifs in the substrate proteins and activates the APC for the ubiquitination of these substrates (Pfleger and Kirschner 2000). To examine whether CDH1 can interact with SnoN through its D box, cell extract was prepared from 293T cells cotransfected with Flag-SnoN and HA-CDH1. SnoN was found to interact with CDH1 (Fig. 6B, lane 4), and this interaction was mediated by the N-terminal half of SnoN (Fig. $6 \mathrm{~B}$, lane 5) and depended on the D box of SnoN as a mutant SnoN lacking the D box did not bind CDH1 (Fig. $6 \mathrm{~B}$, lane 7). In contrast, neither Smad3 nor Smad4 associated with $\mathrm{CDH} 1$ in the same experiment (Fig. 6B). Thus, in the absence of TGF- $\beta$ stimulation, SnoN could be targeted by the APC, albeit with a low affinity, through CDH1, resulting in the low level of constitutive ubiquitination of SnoN (Fig. 1D, lane 4). Consistent with this, a low level of interaction between SnoN and endogenous Cdc27 and Cdc16 was detected even in the absence of TGF- $\beta$ or Smad3 (Fig. 6C, lane 1, top panel).

We next examined whether Smad3, following activation by T $\beta R I$, could recruit additional APC to SnoN to enhance the APC-CDH1-SnoN interaction. Because expression of Smad3 induces degradation of SnoN, which precludes the analysis of SnoN, a proteasome inhibitor, MG132, was used to pretreat the cells to prevent degradation of SnoN. To detect the dependency of Smad3 activity on activation of TGF- $\beta$ signaling, we reduced the expression level of Smad3 so that its nuclear localization and oligomerization activity is dependent on the expression of a constitutively active T $\beta$ RI. In the absence of the active T $\beta R I$, Smad3 associated with the APC components and SnoN in the nucleus (Fig. 6C, lane 3) as shown before, probably because a small portion of transfected Smad3 is located in the nucleus. However, this low level of interaction did not result in any significant recruitment of the APC to SnoN (Fig. 6C, lane 3, top panel). In contrast, in nuclear extract prepared from cells expressing the constitutively active T $\beta \mathrm{RI}$, increased associations between Smad3 and the APC (Fig. 6C, lane 4, second panel from the top) and between Smad3 and SnoN (data not shown) were detected. As a result, a marked increase in the interaction of SnoN with the APC components was observed (Fig. 6C, lane 4, top panel). This increased interaction of SnoN with the APC was mediated by Smad3 because the interaction of the APC with the mutant SnoN (SnoN 89-92A) deficient in binding to Smad3 was not enhanced by Smad3 (Fig. 6C, lanes 7-9), and there was no increase in the APC and SnoN interaction in cells expressing only SnoN and the constitutively active TBRI (Fig. 6C, lane 2). Similarly, Smad2 can also recruit the APC to SnoN in response to TGF- $\beta$ signaling, albeit with a lower efficiency (Fig. 6C, lanes 5,6). Thus, activation of TGF- $\beta$ signaling could result in an increased interaction of $\mathrm{Smad} 3$ and to a lesser extent, Smad2, with the APC and subsequent recruitment of the $\mathrm{APC}$ to SnoN, leading to efficient ubiquitination and degradation of SnoN. When Smads were overexpressed, they were able to recruit the APC to SnoN even in the absence of ligand (data not shown), consistent with the observation that overexpression of Smad2 or Smad3 in $293 \mathrm{~T}$ cells can induce ubiquitination and degradation of SnoN. In TGF- $\beta$-responsive Ba/F3 cells, TGF- $\beta$ also induced the formation of an endogenous Smad3/SnoN/ APC complex (Fig. 6D), again supporting the model that TGF- $\beta$ stimulation results in the recruitment of the APC to SnoN by Smad3. 


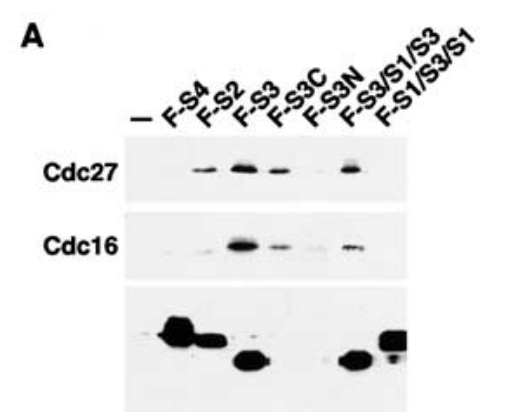

F-Smads
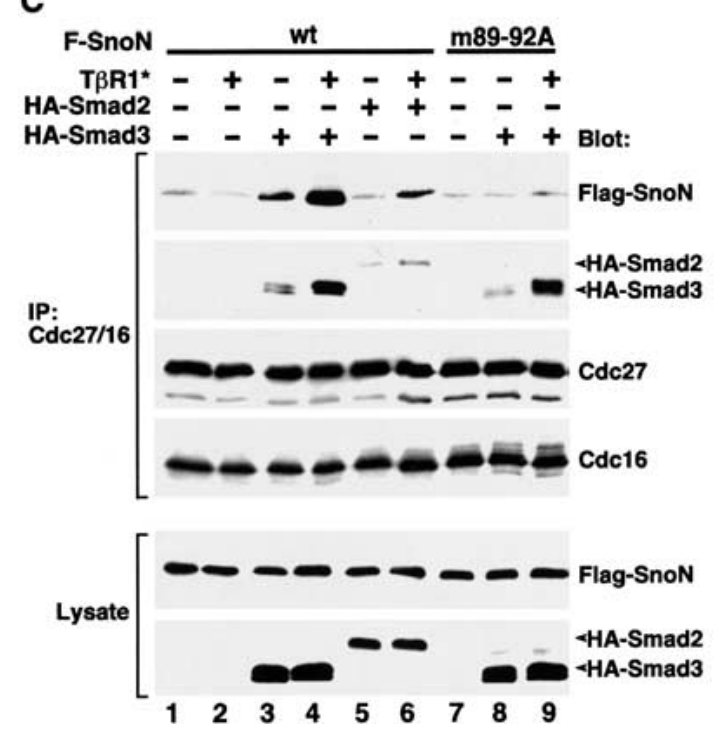

E

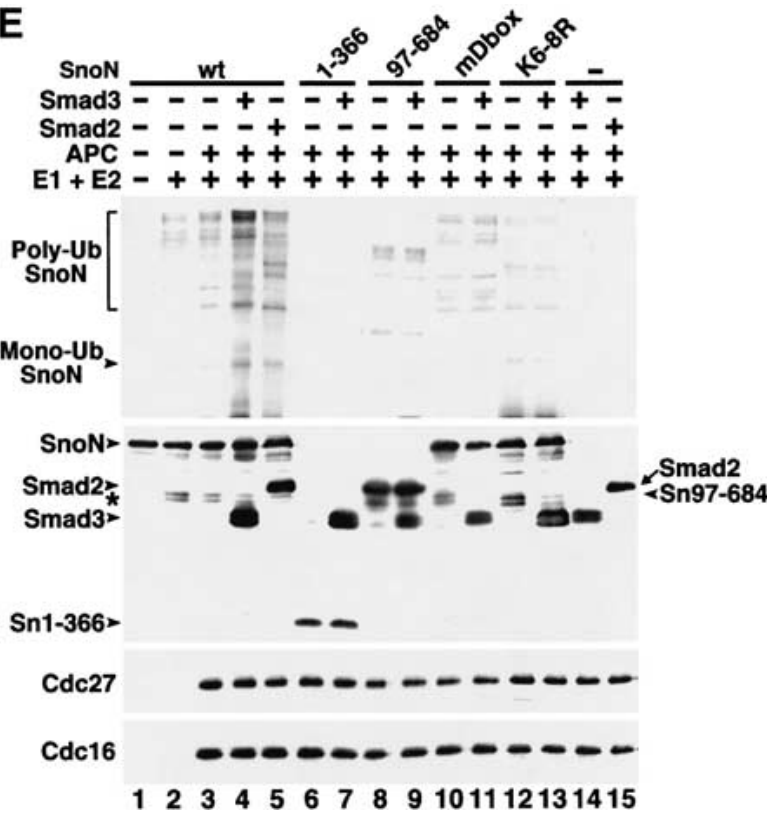

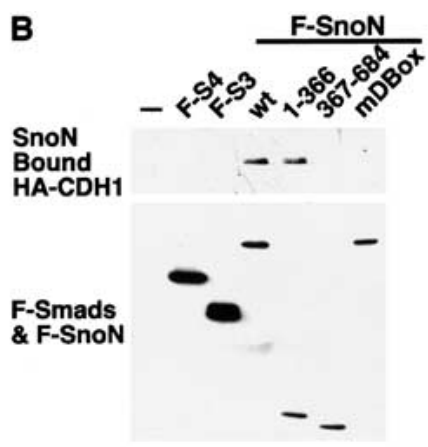

Total HA-CDH1 - - - - -

$\begin{array}{lllllll}1 & 2 & 3 & 4 & 5 & 6 & 7\end{array}$

D

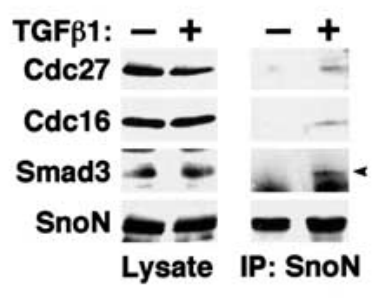

Figure 6. Smad3 and Smad2 interact with the APC to induce ubiquitination of SnoN. (A) Smad3 and Smad2 interact with the APC components. The indicated Flag-tagged full-length or mutant Smads were transfected into 293T cells and isolated from nuclear extracts by immunoprecipitation with anti-Flag agarose. The immunoprecipitates were subjected to immunoblotting with an anti-Cdc27 antibody (top) or anti-Cdc16 (middle) to detect associated APC components, or with anti-Flag to control for Smad levels (bottom). (B) SnoN associates with CDH1 in a $\mathrm{D}$ box-dependent manner. Flag-tagged wild-type or mutant SnoN or Smads were cotransfected with HA-CDH1 into 293T cells and isolated by immunoprecipitation with anti-Flag agarose. The immunoprecipitates were subjected to immunoblotting with anti-HA to detect $\mathrm{CDH} 1$ bound to SnoN (top) and anti-Flag to control for Smad and SnoN levels (middle). Total CDH1 levels were detected by immunoblotting of the cell lysates with anti-HA (bottom). (C) Smad3 or Smad2 recruits the APC to SnoN in response to activation of TGF- $\beta$ signaling. Flag-SnoN and HA-Smad were cotransfected into $293 \mathrm{~T}$ cells in the absence or presence of a constitutive active $\left(T^{204} \mathrm{D}\right) \mathrm{T} \beta \mathrm{RI}\left(\mathrm{T} \beta \mathrm{RI}{ }^{\star}\right)$. The APC was isolated from the nuclear extract by immunoprecipitation with anti-Cdc27 and anti-Cdc16 antisera, and the associated SnoN or Smad3 was detected by immunoblotting with anti-Flag or anti-HA as labeled (two top panels). Total APC levels were detected by immunoblotting with anti-Cdc27 and anti-Cdc16 (third and fourth panels from the top). Total SnoN and Smad3 levels were detected by immunoblotting of the cell lysates with anti-Flag and anti-HA (two bottom panels). (D) TGF- $\beta$ induces formation of an endogenous Smad3/SnoN/APC complex. Nuclear extracts were prepared from Ba/F3 cells that were stimulated with TGF- $\beta$ for 20 min. Smad3 and the APC associated with SnoN was isolated from these nuclear extracts by immunoprecipitation with anti-SnoN and detected by Western blotting with anti-Smad3, anti-Cdc27, or antiCdc16 antibodies. Controls for the levels of total Smad3, SnoN, and APC in the nuclear extracts are shown. $(E)$ In vitro reconstituted ubiquitination reactions. In vitro ubiquitin reactions were carried out with the purified Flag-SnoN, Flag-Smad3 or Flag-Smad2, GST-ubiquitin, and the indicated ubiquitin enzymes as described in Materials and Methods. Ubiquitinated SnoN was detected by Western blotting with anti-SnoN and anti-Flag antisera (top). The membrane was reblotted with anti-Flag (middle) to measure the level of SnoN and Smad3 in each reaction, and with anti-Cdc27 and anti-Cdc16 to determine the amounts of APC in each reaction (two bottom panels). (*) A nonspecific band recognized by anti-SnoN antibody. 
To confirm that the APC can induce ubiquitination of SnoN in a Smad3-dependent manner, an in vitro reconstituted ubiquitination assay was performed with the immunoprecipitated APC from 293T cells and recombinant E1, UbcH5b and UbcH5c (E2), and GST-ubiquitin. Flag-SnoN was purified from transfected $293 \mathrm{~T}$ cells by immunoprecipitation with anti-Flag agarose, eluted with the Flag peptide, and incubated with the above enzymes in vitro in the absence or presence of Smad3. Polyubiquitinated species of SnoN were then detected by immunoblotting with the anti-Flag antibody. As shown in Figure 6E, in the absence of the Smads, a low level of polyubiquitination of SnoN was detected in vitro, dependent on the presence of E1 and E2 (Fig. 6E, lane 2). This low level of constitutive polyubiquitination of SnoN may be attributable to the presence of a low level of endogenous E3 ligase copurified with Flag-SnoN. The addition of purified APC only marginally increased this constitutive ubiquitination (Fig. 6E, lane 3). However, the addition of $\mathrm{CDH} 1$ and the APC significantly enhanced ubiquitination of SnoN (data not shown), suggesting that the APCCDH1 can mediate ubiquitination of SnoN. In a similar reaction, Smad2 or Smad3 themselves were not ubiquitinated by APC (Fig. 6E, lanes 14 and 15), consistent with the observation that $\mathrm{CDH} 1$, which is required for the targeting and activation of the APC, did not interact with Smad3 (Fig. 6B, lane 3). Interestingly, addition of Smad3 and APC in the reaction markedly enhanced the polyubiquitination of SnoN (Fig. 6E, lanes 3,4). This enhancement required the direct interaction between Smad3 and SnoN, because ubiquitination of the SnoN 97-684 mutant that can not bind to Smad3 was not affected by the presence of Smad3 (Fig. 6E, lanes 8,9), and was dependent on the D box, as the ubiquitination of the mDbox was not enhanced by Smad3 (Fig. 6E, lanes 10,11). Consistent with previous observations, SnoN 1-366 that lacks the critical lysine residues required for ubiquitin attachment was not ubiquitinated by APC either in the absence or presence of Smad3 (Fig. 6E, lanes $6,7)$. Although a low level of constitutive ubiquitination of the K6-8R mutant was observed in the presence of the APC, presumably through other neighboring lysine residues, this ubiquitination was not enhanced by Smad3 (Fig. 6E, lanes 12,13), again supporting the model that lysines 440, 446 and 449 are required for TGF- $\beta$ - and Smad3-specific ubiquitination of SnoN. Similar to Smad3, Smad2 also induced ubiquitination of SnoN (Fig. 6E, lane 5). Thus, Smad2 and Smad3 can recruit the APC to SnoN to induce ubiquitination and degradation of SnoN.

\section{Stabilization of SnoN enhances its ability to block TGF- $\beta$ signaling}

If mutation of the lysine ubiquitin attachment sites stabilizes the SnoN protein, these mutants should be more potent than wild-type SnoN in antagonizing TGF- $\beta$ signaling. To test this hypothesis, wild-type SnoN or the SnoN K6-8R mutant were introduced stably into the $\mathrm{Ba} / \mathrm{F} 3$ pro-B cells by retroviral infection, and the pools of infected cells were examined for their ability to respond to TGF- $\beta$-induced growth inhibition. As shown previously, overexpression of wild-type SnoN attenuated the ability of cells to undergo TGF- $\beta$-induced cell cycle arrest only moderately (Stroschein et al. 1999b; Fig. 7B), probably because this molecule is degraded following TGF- $\beta$ stimulation and therefore can not be maintained at a very high level in these cells (Fig. 7A, lane 3). In contrast, SnoN 1-366, which can not be degraded, greatly impaired TGF- $\beta$-induced growth inhibitory response (Stroschein et al. 1999b; Fig. 7A,B). Mutation of the three lysine residues (SnoN K6-8R) impaired SnoN degradation in response to TGF- $\beta$ (Fig. 7A, cf. lane 3 to lane 5) and enhanced the ability of SnoN to attenuate cell-cycle arrest moderately, but reproducibly (Fig. 7B). The fact that SnoN K6-8R did not block TGF- $\beta$ signaling to the same extent as SnoN 1-366 may be attributable to its lower expression level (Fig. 7A). Thus, stabilization of SnoN through mutation of the ubiquitin attachment sites can further enhance attenuation of TGF- $\beta$-induced growth inhibition by SnoN. This again supports that notion that intracellular degradation of SnoN plays an important role in the regulation of TGF- $\beta$ signaling.
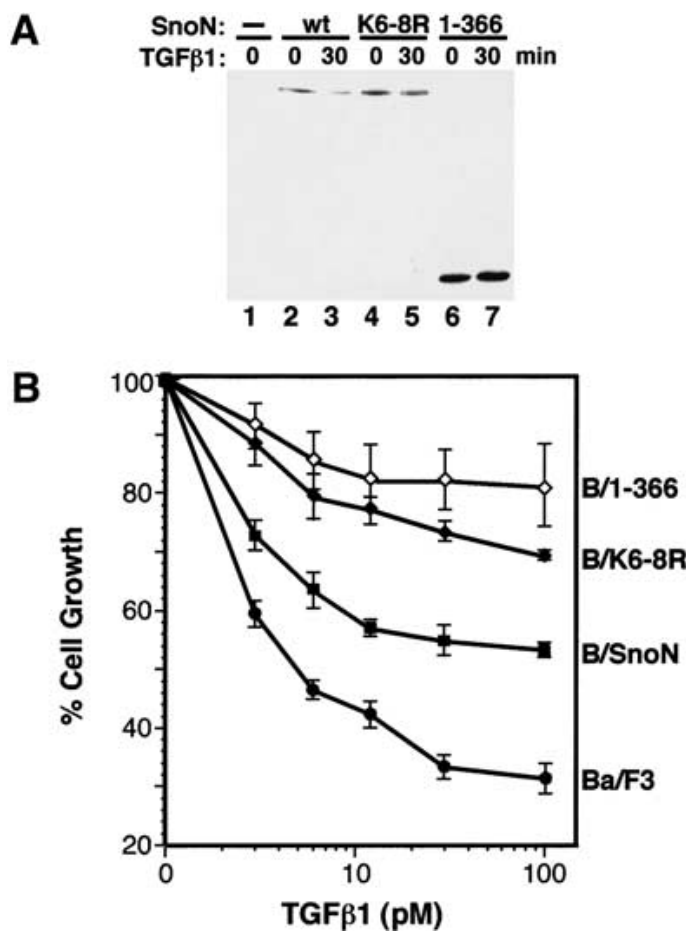

Figure 7. Stabilization of SnoN enhances its ability to block TGF- $\beta$ signaling. (A) Ba/F3 cells stably expressing wild-type SnoN, SnoN 1-366, or SnoN K6-8R were stimulated with (lanes $3,5,7)$ or without (lanes $1,2,4,6)$ TGF- $\beta 1$ for 30 min. Flag-SnoN was isolated by immunoprecipitation with anti-Flag antiserum followed by immunoblotting with anti-Flag. $(B)$ Growth inhibition assay. The same stable Ba/F3 cell lines shown in $A$ were incubated for $4 \mathrm{~d}$ with various concentrations of TGF- $\beta 1$ as indicated. The growth of cells was quantified by cell counting and compared with the growth of unstimulated cells. 


\section{Discussion}

SnoN is an important negative regulator of TGF- $\beta$ signaling that functions to maintain the repressed state of TGF- $\beta$ target genes in the absence of ligand and in the negative feedback control of TGF- $\beta$ signaling. Degradation of SnoN is an essential initial step for the activation of TGF- $\beta$ signaling (Stroschein et al. 1999b). In this report, we have shown that TGF- $\beta$-induced degradation of SnoN requires direct interaction of SnoN with $\operatorname{Smad} 2$ or Smad3, is dependent on the ubiquitin-dependent proteasome, and can be mediated by the APC. At least three regions in SnoN are required for its ubiquitination and degradation in response to TGF- $\beta 1$ (Fig. 8). These include a Smad2/3 binding domain, lysine residues that are ubiquitinated, and the D box that is targeted by the CDH1APC. Mutation of any of the three regions completely blocked TGF- $\beta$ - and Smad3-induced ubiquitination and degradation of SnoN. In the absence of stimuli, SnoN can interact with APC-CDH1 and undergo a low level of constitutive ubiquitination. This may function to maintain the low steady-state level of SnoN in mammalian cells (Fig. 8). However, because the D box in SnoN is not a perfect $\mathrm{D}$ box, its affinity for the APC-CDH1 is low, and this interaction needs to be markedly enhanced by Smad3 or Smad2 to cause efficient ubiquitination of SnoN. These results are consistent with the model (Fig. 8 ) that on activation of TGF- $\beta$ signaling and subsequent nuclear accumulation of the R-Smads, Smad2 or Smad3 interacts with both the APC and SnoN, resulting in recruitment of the APC to SnoN. In the presence of the UbcH5 family of E2 conjugating enzymes, the polyubiquitin chain is then added to the critical lysines 440,446, or 449 of SnoN, leading to its degradation. Consistent with this model, we found that purified APC, together

\section{- TGF $\beta$}
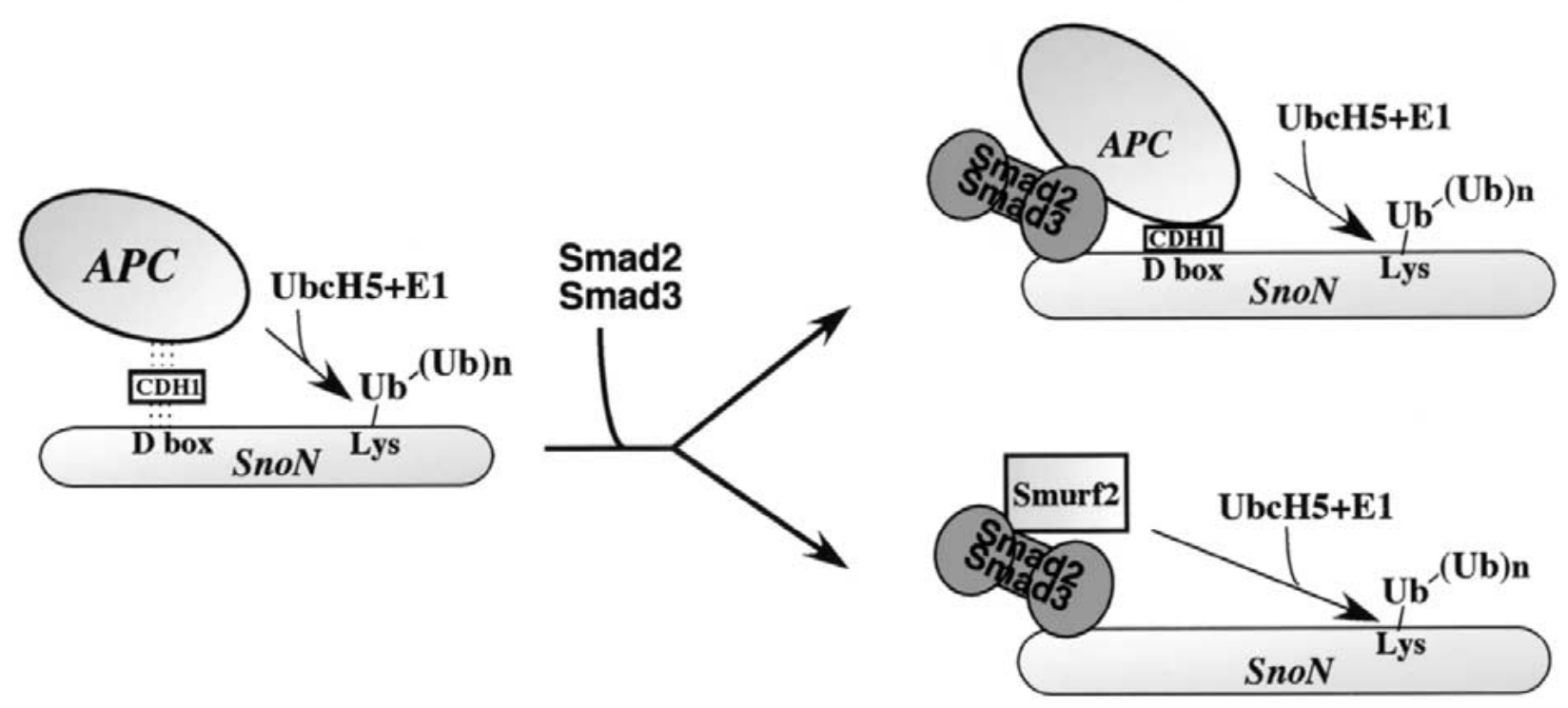

with recombinant $\mathrm{E} 1$ and $\mathrm{UbcH} 5$, can induce polyubiquitination of SnoN in an in vitro reconstituted assay in a R-Smad-dependent manner.

The APC is a multisubunit ubiquitin-protein ligase complex that targets mitotic cyclins and other proteins functioning in late mitosis for degradation, thereby regulating the initiation of anaphase and exit from mitosis (Zachariae and Nasmyth 1999). In addition to these mitosis-related proteins, APC may also degrade proteins that are involved in other important cellular functions in a D box- or KEN box-dependent manner. APC-CDH1 has been shown to induce ubiquitination and degradation of CDC6, a protein critical for the initiation of DNA replication, in a cell cycle-dependent manner (Petersen et al. 2000). Here we provide the first evidence linking the APC to TGF- $\beta$ signaling. In this case, Smad2 and Smad3 are serving as substrate-specific targeting subunits of the APC. It is unclear whether the APC is responsible for the degradation of other TGF- $\beta$ signaling molecules or whether TGF- $\beta$ induces ubiquitination and degradation of other APC substrates. At least in our in vitro reconstituted assays, Smad3 was not ubiquitinated by the APC.

This ability of Smad2 or Smad3 to induce degradation of $\mathrm{SnoN}$ is independent of their ability to heterodimerize with Smad4 because a mutant Smad3 defective in homoand hetero-oligomerization induced SnoN ubiquitination as efficiently as wild-type Smad3 (data not shown). Thus, the population of Smad3 responsible for SnoN degradation may differ from those that complex with Smad4 to regulate transcriptional activation. This is consistent with the observation that Smad3 is present in many different protein complexes of various sizes before and after TGF- $\beta$ signaling (Jayaraman and Massague 2000). Inter-

Figure 8. Model of ubiquitination and degradation of SnoN. 
estingly, although Smad3 can interact with the APC component to induce degradation of SnoN, its own stability was not affected by the APC (data not shown). Because Smad2 and Smad3 have been shown to function in concert with the HECT-domain ubiquitin ligase, Smurf2 (Bonni et al. 2001; data not shown), and with the APC to induce degradation of SnoN, at least two ubiquitination pathways can be activated by TGF- $\beta$ to induce degradation of SnoN. The observation that Smad3 $\Delta \mathrm{PY}$, deficient in the recruitment of Smurf2, was less efficient than wild-type Smad3, but nevertheless was capable of mediating the degradation of SnoN (Fig. 5B), suggests that both the Smurf2 pathway and the APC pathway are required for maximal degradation of SnoN by the Smads. These two pathways may function either synergistically or differentially in a tissue type- or developmental stagespecific manner to regulate SnoN level and TGF- $\beta$ responses. The involvement of multiple ubiquitination pathways has also been demonstrated for the degradation of $\beta$-catenin (Polakis 2001). Future experiments will investigate the contribution of each pathway in regulation of SnoN expression.

Our results using the dominant-negative mutants of various E2 enzymes suggest the involvement of the UbcH5 family of enzymes in TGF- $\beta$-induced degradation of SnoN. UbcH5 belongs to an evolutionarily conserved subfamily of E2s that are highly homologous to yeast UBC4/UBC5 (Scheffner et al. 1994; Jensen et al. 1995; Rolfe et al. 1995). This family of ubiquitin-conjugating enzymes has been shown to function in concert with the HECT-domain E3 ligases as well as the APC in vitro. In reconstituted ubiquitination assays, UbcH5b (hUBC4) can complex with the HECT-domain ligase, E6-AP, to stimulate ubiquitination of p53 (Scheffner et al. 1994; Jensen et al. 1995; Rolfe et al. 1995) or together with APC11, the RING-finger containing subunit of APC, to induce polyubiquitination of cyclin B or securin (Gmachl et al. 2000; Leverson et al. 2000). These functional capacities of UbcH5 are consistent with our findings that both the APC and Smurf2 may be involved in the degradation of SnoN. In addition to SnoN, UbcH5b and $\mathrm{UbcH} 5 \mathrm{c}$ have been found to mediate degradation of Smad2 in an activation-dependent manner (Lo and Massague 1999). It is not clear which E3 ligase is involved in that process. Another E2, UbcH10 (E2-C), has also been implicated in APC-mediated ubiquitination (Aristarkhov et al. 1996; Townsley et al. 1997), but a dominantnegative form of this E2 had no effect on Smad3-induced degradation of SnoN (Fig. 6A). The three members of $\mathrm{UbcH} 5, \mathrm{UbcH} 5 \mathrm{a}, \mathrm{UbcH} 5 \mathrm{~b}$, and $\mathrm{UbcH} 5 \mathrm{c}$, are highly related $188 \%$ identity between $\mathrm{UbcH} 5 \mathrm{a}$ and $\mathrm{UbcH} 5 \mathrm{~b}$ or $\mathrm{UbcH} 5 \mathrm{c}$ and $97 \%$ identity between UbcH5b and $\mathrm{UbcH} 5 \mathrm{c})$. It is not clear whether these proteins display any functional specificity in their ability to mediate ubiquitination of SnoN in vivo.

We found that three closely positioned lysine residues in SnoN, Lys 440, Lys 446, and Lys 449 are required for ubiquitination by both the APC (Fig. 4C) and Smurf2 (data not shown). Mutation of these residues either individually or in combination greatly impaired the ubiqui- tination of SnoN and resulted in stabilization of SnoN (Fig. 7A) and in enhancement of the ability of SnoN to block TGF- $\beta$ signaling (Fig. 7B). Thus, it is likely that the three lysines, together with the neighboring amino acid residues, form a structure determinant that allows transfer of the ubiquitin moiety to one or more of the three lysines. Alternatively, these lysine residues may function in a cooperative manner to facilitate ubiquitin attachment. SnoN has two alternatively spliced forms, SnoI (399 amino acids) and SnoA (425 amino acids) that share only the first 366 amino acid residues with SnoN (Pearson-White 1993). They interact with Smad2 and Smad3 and contain the D box, but lack the three lysine residues attributable to truncation of the molecules by alternative splicing. We found that SnoN 1-366 can not be degraded in response to TGF- $\beta$ stimulation and is more potent in rendering cells resistant to TGF- $\beta$-induced growth inhibition. Indeed, SnoI has been detected in rhabdomyosarcoma tumor (Pearson-White 1993), suggesting that stabilization of SnoN may contribute to the transforming activity of SnoN.

Deregulation of protein degradation can lead to tumorigenesis through misregulated levels of critical oncogene products or tumor suppressor proteins. The transforming oncoprotein v-Jun, a truncated version of c-Jun, lacks a 27 amino acid domain required for Jun degradation (Treier et al. 1994). The lack of degradation results in an increased cellular level of v-Jun, and this may contribute to its transforming ability. The $\mathrm{G}_{1} \mathrm{CDK}$ inhibitor p27Kip1 is found in decreased levels in aggressive colorectal (Loda et al. 1997) and breast carcinomas (Catzavelos et al. 1997) because of increased degradation of p27. SnoN was originally defined as an oncogene because high levels of wild-type SnoN lead to transformation of chicken and quail embryo fibroblasts (Boyer et al. 1993). High levels of SnoN are also detected in lymphomas and in carcinoma cells of the stomach, thyroid, and lung (Nomura et al. 1989). It is still not clear how the expression level of SnoN is elevated in cancer cells. Our current study has revealed the cellular mechanism and pathways for the regulation of SnoN degradation and provides a potential mechanism for the induction of oncogenic potential of SnoN.

\section{Materials and methods}

Cells, antisera, and constructs

Phoenix-Eco and 293T cells were maintained in Dulbecco's modified eagle medium (DMEM) supplemented with 10\% fetal bovine serum (FBS). The Hep3B human hepatoma cells (ATCC) were maintained in minimal essential medium (MEM) supplemented with $10 \%$ FBS. The Ba/F3 pro-B cells were grown in RPMI supplemented with $10 \%$ FBS and $10 \%$ WEHI cell-conditioned medium as a source of interleukin-3 (Luo and Lodish 1996).

Antisera against ubiquitin (FL-76), SnoN (H-317), Smad3 (FL425), Cdc27 (C-20), and Cdc16 (K-16) were purchased from Santa Cruz Biotechnology. Antiserum against the Flag peptide was purchased from Sigma. Two polyclonal antibodies against SnoN were described previously (Stroschein et al. 1999b). Smad, 
SnoN, CDH1, and dominant negative E2 (UbcH6, UbcH7, $\mathrm{UbcH} 8$, and $\mathrm{UbcH} 9$ ) mutants were generated by PCR and cloned into pCMV5b. Dominant-negative E2 mutations: UbcH5a, C85A; UbcH5b, C85A; UbcH5c, C85A; UbcH6, C131A; UbcH7, C86A; UbcH8, C85A; UbcH9, C93A; and UbcH10, C114S. Smad3 $\triangle$ PY: lacking residues $180-184$.

\section{Transfection and retroviral infection}

Phoenix-Eco, 293T, and Hep3B cells were transiently transfected using lipofectamine-plus (GIBCO Life Technologies). To generate stable $\mathrm{Ba} / \mathrm{F} 3$ cell lines expressing wild-type or mutated SnoN, Flag-SnoN in the retroviral vector pMX-IRES-GFP (Liu et al. 1997b) that also expresses the green fluorescent protein (GFP) was used to transfect Phoenix-Eco cells. Forty-eight hours after transfection, $5 \times 105 \mathrm{Ba} / \mathrm{F} 3$ cells were cocultivated with the transfected Phoenix-Eco cells for $24 \mathrm{~h}$, and the infected cells were selected by cell sorting on the basis of GFP expression.

\section{Immunoprecipitation and Western blotting}

Flag- and/or HA-tagged Smads and SnoN were isolated from transfected $293 \mathrm{~T}$ cells by immunoprecipitation with anti-Flag (Sigma) or anti-HA agarose, followed by elution with Flag or HA peptide as described previously (Zhou et al. 1998a; Stroschein et al. 1999a). To detect endogenous APC associated with the Smads, nuclear extracts were prepared from 293T cells transfected with Flag-tagged Smad as described previously (Lee et al. 1987), and the Flag-tagged protein was isolated by immunoprecipitation with anti-Flag agarose (Sigma), followed by elution with the Flag peptide as described previously (Zhou et al. 1998a; Stroschein et al. 1999a). Associated Cdc27 and Cdc16 were detected by Western blotting with anti-Cdc27 and anti-Cdc16. To detect SnoN and Smad3 associated with the APC, nuclear extracts were prepared from $293 \mathrm{~T}$ cells transfected with Flagtagged SnoN and HA-tagged Smad3. The APC was isolated by immunoprecipitation with anti-Cdc27 and anti-Cdc16, and the associated Smad3 and SnoN were detected by Western blotting with anti-HA and anti-Flag antibodies. To detect endogenous Smad3 and SnoN associated with the APC, nuclear extracts were prepared from $\mathrm{Ba} / \mathrm{F} 3$ cells treated for 0 or 20 min with TGF- $\beta 1$. SnoN was isolated by immunoprecipitation with antiSnoN, and the associated Smad3 and APC were detected by Western blotting with anti-Smad3, anti-Cdc27, and anti-Cdc16 antibodies. Immunoprecipitation of endogenous SnoN from Hep3B cells was performed as described previously (Stroschein et al. 1999b).

To measure TGF- $\beta$-induced degradation of SnoN in Ba/F3 cells, $2 \times 107 \mathrm{Ba} / \mathrm{F} 3$ cells were serum-starved for $15 \mathrm{~h}$ and treated with TGF- $\beta 1$ for 0 or 30 min. Nuclear extracts were prepared, and Flag-SnoN was detected by Western blotting with anti-Flag.

To detect ubiquitination of SnoN in vivo, Flag-SnoN, either singly or cotransfected with Smads, was isolated from 293T cells by immunoprecipitation with the anti-Flag agarose followed by elution with the Flag peptide. After equalizing the amounts of SnoN in each sample, the levels of SnoN ubiquitination were measured by Western blotting with an anti-ubiquitin antiserum (Santa Cruz).

\section{Growth inhibition assay}

For growth inhibition assay, $1 \times 104 \mathrm{Ba} / \mathrm{F} 3$ cells were incubated with various concentrations of TGF- $\beta 1$ for 3-4 $\mathrm{d}$. The growth of cells was determined by cell counting and compared with that of unstimulated cells (Luo and Lodish 1996). The assay was set up in triplicate, and standard errors are shown.

\section{Pulse-chase assays}

Transfected 293T cells were pulse-labeled for $30 \mathrm{~min}$ in the presence of ${ }^{35} \mathrm{~S}$-express $(0.25 \mathrm{mCi} / \mathrm{ml}, \mathrm{NEN})$ and chased for various periods of time. SnoN was purified by immunoprecipitation and resolved on an SDS-polyacrylamide gel. For treatments with proteasome inhibitors, transfected cells were pretreated with $10 \mu \mathrm{M}$ lactacystin (Calbiochem), $25 \mu \mathrm{M}$ MG132 (Calbiochem), or $25 \mu \mathrm{M}$ N-acetyl-Leu-Leu-Methioninal (ALLM) (Sigma) for $45 \mathrm{~min}$ and then throughout the duration of the pulse and chase.

\section{In vitro ubiquitination assay}

Standard ubiquitination assays were carried out in $15 \mu \mathrm{L}$ of QA (20 mM Tris- $\mathrm{HCl}$ at $\mathrm{pH} 7.7,100 \mathrm{mM} \mathrm{KCl}, 0.1 \mathrm{mM} \mathrm{CaCl}_{2}, 1 \mathrm{mM}$ $\mathrm{MgCl}_{2}, 1 \mathrm{mM}$ DTT) (King et al. 1995; Gmachl et al. 2000) containing $15 \mu \mathrm{g}$ of GST-ubiquitin, $1 \mu \mathrm{g}$ of E1 (Boston Biochem), 1.5 $\mu \mathrm{g}$ of E2 (GST-UbcH5b and UbcH5c), $0.6 \mu \mathrm{L}$ of an ATP-regenerating system and Flag-SnoN and/or Flag-Smad3 or Flag-Smad2 immunopurified from 293 T cells. The APC complex was immunoprecipitated from $293 \mathrm{~T}$ cells with antisera against Cdc27 and Cdc16 (Santa Cruz) and added to the reaction while still immobilized on the Sepharose beads. Reactions were incubated for $30 \mathrm{~min}$ at $37^{\circ} \mathrm{C}$ and terminated by the addition of SDS-sample buffer. Polyubiquitinated substrates were detected by Western blotting with anti-Flag and anti-SnoN antibodies.

\section{Acknowledgments}

We thank Drs S. Pearson-White for the SnoN cDNA, M. Scheffner for the UbcH6 and UbcH7 cDNAs and GST-ubiquitin, K. Iwai for the dominant-negative $\mathrm{UbcH} 5 \mathrm{a}, \mathrm{UbcH} 5 \mathrm{~b}$, and $\mathrm{UbcH} 5 \mathrm{c}$ cDNAs, P. Howley for the UbcH8 and UbcH9 cDNAs, J. Ruderman for the dominant negative UbcH10 cDNA, and J-M. Peters for the CDH1 cDNA. We wish to thank Marc Kirschner for drawing our attention to the D box and for communication of unpublished results on APC and CDH1. We are also grateful to J. He for mapping and construction of SnoN 89-92A, to B. Krechman for construction of several HA-tagged SnoN lysine mutants and to Q. Zhou for critical reading of the manuscript. This work was supported by NIH grant CA87940, the California TRDRP grant 9RT-154 and American Cancer Society Research Scholar's Award to K. Luo. S.L.S was supported by predoctoral fellowships from the National Science Foundation and Department of Defense Breast Cancer Research Program.

The publication costs of this article were defrayed in part by payment of page charges. This article must therefore be hereby marked "advertisement" in accordance with 18 USC section 1734 solely to indicate this fact.

\section{References}

Akiyoshi, S., Inoue, H., Hanai, J., Kusanagi, K., Nemoto, N., Miyazono, K., and Kawabata, M. 1999. c-Ski acts as a transcriptional co-repressor in transforming growth factor-beta signaling through interaction with smads. I. Biol. Chem. 274: 35269-35277.

Aristarkhov, A., Eytan, E., Moghe, A., Admon, A., Hershko, A., and Ruderman, J.V. 1996. E2-C, a cyclin-selective ubiquitin carrier protein required for the destruction of mitotic cyclins. Proc. Natl. Acad. Sci. 93: 4294-4299. 
Bonni, S., Wang, H.-R., Causing, C.G., Kavsak, P., Stroschein, S.L., Luo, K., and Wrana, J.L. 2001. TGF- $\beta$ induces assembly of a Smad2-Smurf2 ubiquitin ligase complex that targets SnoN for degradation. Nat. Cell. Biol. 3: 587-595.

Boyer, P.L., Colmenares, C., Stavnezer, E., and Hughes, S.H. 1993. Sequence and biological activity of chicken snoN cDNA clones. Oncogene 8: 457-466.

Catzavelos, C., Bhattacharya, N., Ung, Y.C., Wilson, J.A., Roncari, L., Sandhu, C., Shaw, P., Yeger, H., Morava-Protzner, I., Kapusta, L., et al. 1997. Decreased levels of the cell-cycle inhibitor p27Kip1 protein: Prognostic implications in primary breast cancer. Nat. Med. 3: 227-230.

Chacko, B.M., Qin, B., Correia, J.J., Lam, S.S., de Caestecker, M.P., and Lin, K. 2001. The L3 loop and C-terminal phosphorylation jointly define Smad protein trimerization. Nat. Struct. Biol. 8: 248-253.

Chen, X., Rubock, M.J., and Whitman, M. 1996. A transcriptional partner for MAD proteins in TGF- $\beta$ signalling. Nature 383: 691-696.

Chen, X., Weisberg, E., Fridmacher, V., Watanabe, M., Naco, G., and Whitman, M. 1997. Smad4 and FAST-1 in the assembly of activin-responsive factor. Nature 389: 85-89.

Colmenares, C. and Stavnezer, E. 1989. The ski oncogene induces muscle differentiation in quail embryo cells. Cell 59: 293-303.

Colmenares, C., Sutrave, P., Hughes, S.H., and Stavnezer, E. 1991. Activation of the c-ski oncogene by overexpression. J. Virol. 65: 4929-4935.

Coux, O., Tanaka, K., and Goldberg, A.L. 1996. Structure and functions of the 20S and 26S proteasomes. Annu. Rev. Biochem. 65: 801-847.

Fang, G., Yu, H., and Kirschner, M.W. 1998. Direct binding of CDC20 protein family members activates the anaphasepromoting complex in mitosis and G1. Mol. Cell 2: 163 171.

Fumagalli, S., Doneda, L., Nomura, N., and Larizza, L. 1993. Expression of the c-ski proto-oncogene in human melanoma cell lines. Melanoma Res. 3: 23-27.

Germain, S., Howell, M., Esslemont, G.M., and Hill, C.S. 2000. Homeodomain and winged-helix transcription factors recruit activated Smads to distinct promoter elements via a common Smad interaction motif. Genes \& Dev. 14: 435451.

Gieffers, C., Peters, B.H., Kramer, E.R., Dotti, C.G., and Peters, J.M. 1999. Expression of the CDH1-associated form of the anaphase-promoting complex in postmitotic neurons. Proc. Natl. Acad. Sci. 96: 11317-11322.

Glotzer, M., Murray, A.W., and Kirschner, M.W. 1991. Cyclin is degraded by the ubiquitin pathway. Nature 349: 132-138.

Gmachl, M., Gieffers, C., Podtelejnikov, A.V., Mann, M., and Peters, J.M. 2000. The RING-H2 finger protein APC11 and the E2 enzyme UBC4 are sufficient to ubiquitinate substrates of the anaphase-promoting complex. Proc. Natl. Acad. Sci. 97: 8973-8978.

Gonen, H., Bercovich, B., Orian, A., Carrano, A., Takizawa, C., Yamanaka, K., Pagano, M., Iwai, K., and Ciechanover, A. 1999. Identification of the ubiquitin carrier proteins, E2s, involved in signal-induced conjugation and subsequent degradation of ІкB $\alpha$. J. Biol. Chem. 274: 14823-14830.

Heldin, C.-H., Miyazono, K., and ten Dijke, P. 1997. TGF- $\beta$ signalling from cell membrane to nucleus through SMAD proteins. Nature 390: 465-471.

Hershko, A. and Ciechanover, A. 1998. The ubiquitin system. Annu. Rev. Biochem. 67: 425-479.

Huibregtse, J.M., Scheffner, M., Beaudenon, S., and Howley, P.M. 1995. A family of proteins structurally and functionally related to the E6-AP ubiquitin-protein ligase. Proc. Natl. Acad. Sci. 92: 5249.

Jayaraman, L. and Massague, J. 2000. Distinct oligomeric states of SMAD proteins in the transforming growth factor-beta pathway. J. Biol. Chem. 275: 40710-40717.

Jensen, J.P., Bates, P.W., Yang, M., Vierstra, R.D., and Weissman, A.M. 1995. Identification of a family of closely related human ubiquitin conjugating enzymes. J. Biol. Chem. 270: 30408-30414.

Kavsak, P., Rasmussen, R.K., Causing, C.G., Bonni, S., Zhu, H., Thomsen, G.H., and Wrana, J.L 2000. Smad7 binds to Smurf2 to form an E3 ubiquitin ligase that targets the TGF- $\beta$ receptor for degradation. Mol. Cell 6: 1365-1375.

Kim, R.H., Wang, D., Tsang, M., Martin, J., Huff, C., de Caestecker, M.P., Parks, W.T., Meng, X., Lechleider, R.J., Wang, T., and Roberts, A.B. 2000. A novel smad nuclear interacting protein, SNIP1, suppresses p300-dependent TGF- $\beta$ signal transduction. Genes \& Dev. 14: 1605-1616.

King, R.W., Peters, J.M., Tugendreich, S., Rolfe, M., Hieter, P., and Kirschner, M.W. 1995. A 20S complex containing CDC27 and CDC16 catalyzes the mitosis-specific conjugation of ubiquitin to cyclin B. Cell 81: 279-288.

Kramer, E.R., Scheuringer, N., Podtelejnikov, A.V., Mann, M., and Peters, J.M. 2000. Mitotic regulation of the APC activator proteins CDC20 and CDH1. Mol. Biol. Cell 11: 15551569.

Labbe, E., Silvestri, C., Hoodless, P.A., Wrana, J.L., and Attisano, L. 1998. Smad2 and Smad3 positively and negatively regulate TGF- $\beta$-dependent transcription through the forkhead DNA-binding protein FAST2. Mol. Cell 2: 109120.

Lee, K.A.W., Bindereif, A., and Green, M.R. 1987. A small-scale procedure for preparation of nuclear extracts that support efficient transcription and pre-mRNA splicing. Gene Anal. Tech.. 5: 22-31.

Leverson, J.D., Joazeiro, C.A., Page, A.M., Huang, H., Hieter, P., and Hunter, T. 2000. The APC11 RING-H2 finger mediates E2-dependent ubiquitination. Mol. Biol. Cell 11: 23152325.

Lin, X., Liang, M., and Feng, X.H. 2000. Smurf2 is a ubiquitin E3 ligase mediating proteasome-dependent degradation of Smad2 in transforming growth factor-beta signaling. J. Biol. Chem. 275: 36818-36822.

Liu, F., Pouponnot, C., and Massague, J. 1997a. Dual role of the Smad4/DPC4 tumor suppressor in TGF- $\beta$-inducible transcriptional complexes. Genes \& Dev. 11: 3157-3167.

Liu, X., Sun, Y., Constantinescu, S.N., Karam, E., Weinberg, R.A., and Lodish, H.F. 1997b. Transforming growth factor beta-induced phosphorylation of Smad3 is required for growth inhibition and transcriptional induction in epithelial cells. Proc. Nat1. Acad. Sci. 94: 10669-10674.

Lo, R.S. and Massague, J. 1999. Ubiquitin-dependent degradation of TGF- $\beta$-activated smad2. Nat. Cell. Biol. 1: 472478.

Loda, M., Cukor, B., Tam, S.W., Lavin, P., Fiorentino, M., Draetta, G.F., Jessup, J.M., and Pagano, M. 1997. Increased proteasome-dependent degradation of the cyclin-dependent kinase inhibitor p27 in aggressive colorectal carcinomas. Nat. Med. 3: 231-234.

Lorca, T., Castro, A., Martinez, A.M., Vigneron, S., Morin, N., Sigrist, S., Lehner, C., Doree, M., and Labbe, J.C. 1998. Fizzy is required for activation of the APC/cyclosome in Xenopus egg extracts. EMBO J. 17: 3565-3575.

Luo, K. and Lodish, H.F. 1996. Signaling by chimeric erythropoietin-TGF- $\beta$ receptors: Homodimerization of the cytoplasmic domain of the type I TGF- $\beta$ receptor and heterodimer- 
ization with the type II receptor are both required for intracellular signal transduction. EMBO J. 15: 4485-4496.

Luo, K., Stroschein, S.L., Wang, W., Chen, D., Martens, E., Zhou, S., and Zhou, Q. 1999. The Ski oncoprotein interacts with the Smad proteins to repress TGF- $\beta$ signaling. Genes \& Dev. 13: 2196-2206.

Massague, J. and Wotton, D. 2000. Transcriptional control by the TGF- $\beta /$ Smad signaling system. EMBO J. 19: 1745-1754.

Massague, J., Blain, S.W., and Lo, R.S. 2000. TGF- $\beta$ signaling in growth control, cancer, and heritable disorders. Cell 103: 295-309.

Nomura, N., Sasamoto, S., Ishii, S., Date, T., Matsui, M., and Ishizaki, R. 1989. Isolation of human cDNA clones of ski and the ski-related gene, sno. Nucleic Acids Res. 17: 5489-5500.

Pearson-White, S. 1993. SnoI, a novel alternatively spliced isoform of the ski protooncogene homolog, sno. Nucleic Acids Res. 21: 4632-4638.

Peters, J.M. 1999. Subunits and substrates of the anaphase-promoting complex. Exp. Cell Res. 248: 339-349.

Petersen, B.O., Wagener, C., Marinoni, F., Kramer, E.R., Melixetian, M., Denchi, E.L., Gieffers, C., Matteucci, C., Peters, J.M., and Helin, K. 2000. Cell cycle- and cell growth-regulated proteolysis of mammalian CDC6 is dependent on APC-CDH1. Genes \& Dev. 14: 2330-2343.

Pfleger, C.M. and Kirschner, M.W. 2000. The KEN box: An APC recognition signal distinct from the D box targeted by Cdh1. Genes \& Dev. 14: 655-665.

Polakis, P. 2001. More than one way to skin a catenin. Cell 105: 563-566.

Rolfe, M., Beer-Romero, P., Glass, S., Eckstein, J., Berdo, I., Theodoras, A., Pagano, M., and Draetta, G. 1995. Reconstitution of p53-ubiquitinylation reactions from purified components: The role of human ubiquitin-conjugating enzyme UBC4 and E6-associated protein (E6AP). Proc. Natl. Acad. Sci. 92: 3264-3268.

Rudner, A.D. and Murray, A.W. 2000. Phosphorylation by Cdc28 activates the Cdc20-dependent activity of the anaphase-promoting complex. J. Cell. Biol. 149: 1377-1390.

Scheffner, M., Huibregtse, J.M., Vierstra, R.D., and Howley, P.M. 1993. The HPV-16 E6 and E6-AP complex functions as a ubiquitin-protein ligase in the ubiquitination of p53. Cell 75: 495-505.

Scheffner, M., Huibregtse, J.M., and Howley, P.M. 1994. Identification of a human ubiquitin-conjugating enzyme that mediates the E6-AP-dependent ubiquitination of p53. Proc. Nat1. Acad. Sci. 91: 8797-8801.

Shinagawa, T., Dong, H.-D., Xu, M., Maekawa, T., and Ishii, S. 2000. The sno gene, which encodes a component of the histone deacetylation complex, acts as a tumor suppressor in mice. EMBO J. 19: 2280-2291.

Shirayama, M., Toth, A., Galova, M., and Nasmyth, K. 1999. $\mathrm{APC}(\mathrm{Cdc} 20)$ promotes exit from mitosis by destroying the anaphase inhibitor Pds1 and cyclin Clb5. Nature 402: 203 207.

Stroschein, S.L., Wang, W., and Luo, K. 1999a. Cooperative binding of Smad proteins to two adjacent DNA elements in the plasminogen activator inhibitor-1 promoter mediates transforming growth factor $\beta$-induced Smad-dependent transcriptional activation. J. Biol. Chem. 274: 9431-9441.

Stroschein, S.L., Wang, W., Zhou, S., Zhou, Q., and Luo, K. $1999 \mathrm{~b}$. Negative feedback regulation of TGF- $\beta$ signaling by the SnoN oncoprotein. Science 286: 771-774.

Sun, Y., Liu, X., Eaton, E.N., Lane, W.S., Lodish, H.F., and Weinberg, R.A. 1999a. Interaction of the Ski oncoprotein with Smad3 regulates TGF- $\beta$ signaling. Mol. Cell 4: 499-509.
Sun, Y., Liu, X., Ng-Eaton, E., Lodish, H.F., and Weinberg, R.A. 1999b. SnoN and Ski protooncoproteins are rapidly degraded in response to transforming growth factor beta signaling. Proc. Natl. Acad. Sci. 96: 12442-12447.

Townsley, F.M., Aristarkhov, A., Beck, S., Hershko, A., and Ruderman, J.V. 1997. Dominant-negative cyclin-selective ubiquitin carrier protein E2-C/UbcH10 blocks cells in metaphase. Proc. Nat1. Acad. Sci. 94: 2362-2367.

Treier, M., Staszewski, L.M., and Bohmann, D. 1994. Ubiquitindependent c-Jun degradation in vivo is mediated by the delta domain. Cell 78: 787-798.

Varshavsky, A. 1997. The ubiquitin system. Trends Biochem. Sci. 22: 383-387.

Wotton, D., Lo, R.S., Lee, S., and Massague, J. 1999. A Smad transcriptional corepressor. Cell 97: 29-39.

Wu, J.W., Fairman, R., Penry, J., and Shi, Y. 2001. Formation of a stable hetero-dimer between Smad2 and Smad4. I. Biol. Chem. 276: 20688-20694.

Wu, R.Y., Zhang, Y., Feng, X.H., and Derynck, R. 1997. Heteromeric and homomeric interactions correlate with signaling activity and functional cooperativity of Smad3 and Smad4/ DPC4. Mol. Cell. Biol. 17: 2521-2528.

Zachariae, W. and Nasmyth, K. 1999. Whose end is destruction: Cell division and the anaphase-promoting complex. Genes \& Dev. 13: 2039-2058.

Zhang, Y., Musci, T., and Derynck, R. 1997. The tumor suppressor Smad4/DPC 4 as a central mediator of Smad function. Curr. Biol. 7: 270-276.

Zhang, Y., Chang, C., Gehling, D.J., Hemmati-Brivanlou, A., and Derynck, R. 2001. Regulation of Smad degradation and activity by Smurf2, an E3 ubiquitin ligase. Proc. Nat1. Acad. Sci. 98: 974-979.

Zhou, Q., Chen, D., Pierstorff, E., and Luo, K. 1998a. Transcription elongation factor P-TEF $\beta$ mediates Tat activation of HIV-1 transcription at multiple stages. EMBO J. 17:36813691.

Zhou, S., Zawel, L., Lengauer, C., Kinzler, K.W., and Vogelstein, B. 1998b. Characterization of human FAST-1, a TGF- $\beta$ and activin signal transducer. Mol. Cell 2: 121-127.

Zhu, H., Kavsak, P., Abdollah, S., Wrana, J.L., and Thomsen, G.H. 1999. A SMAD ubiquitin ligase targets the BMP pathway and affects embryonic pattern formation. Nature 400: $687-693$ 


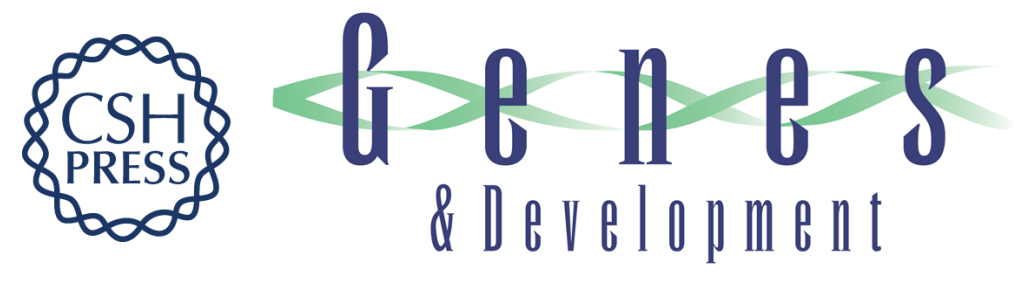

\section{Smad3 recruits the anaphase-promoting complex for ubiquitination and degradation of SnoN}

Shannon L. Stroschein, Shirin Bonni, Jeffrey L. Wrana, et al.

Genes Dev. 2001, 15:

Access the most recent version at doi:10.1101/gad.912901

References This article cites 67 articles, 33 of which can be accessed free at: http://genesdev.cshlp.org/content/15/21/2822.full.html\#ref-list-1

License

Email Alerting

Receive free email alerts when new articles cite this article - sign up in the box at the top Service right corner of the article or click here.

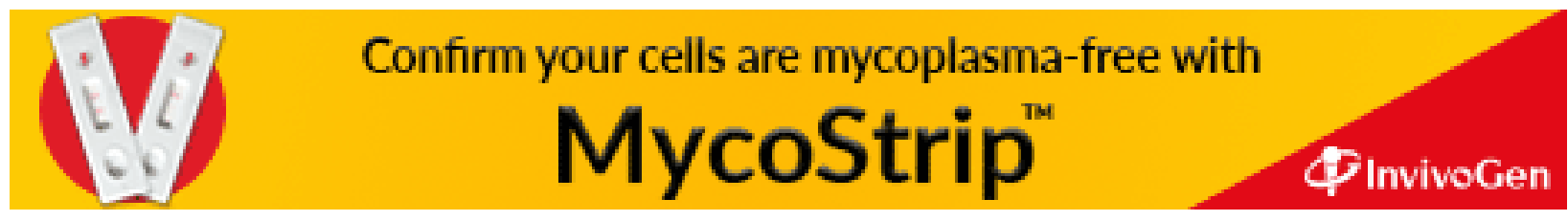

